\title{
¿ES ALCANZABLE EL OBJETIVO FINANCIERO DE MAXIMIZAR EL VALOR A TRAVÉS DE LA INVERSIÓN EN LA BOLSA MEXICANA DE VALORES?
}

\section{Is it Achievable the Financial Objective of Maximizing the Value by Means of Investing in the Mexican Stock Exchange?}

\author{
Rosa María Ortega Ochoa ${ }^{1}$ \\ Raúl Arturo Cornejo López²(†) \\ Eduardo Villegas Hernández ${ }^{3}$
}

\section{RESUMEN}

En este trabajo buscamos dar cuenta de la importancia de las inversiones, considerando que el propósito de cualquier inversionista es maximizar su valor. El tema es relevante para todo aquellos que invierten en la BMV y en especial para las pensiones de los adultos mayores, tema que cruza la atención de gobiernos, iniciativa privada, trabajadores y organizaciones sin fines de lucro, ya que quizá sea la única forma de evitar una futura población de adultos mayores en situación de pobreza. Se trata de que sea garantizado un flujo adecuado de los ingresos cuando se suspenden por vejez, invalidez o riesgos de trabajo. Por tanto, debe administrarse adecuadamente la inversión que los trabajadores realizan a través de las Siefores. Importa ver si la inversión en la BMV

\footnotetext{
${ }^{1}$ Maestra en Finanzas, FCA-UNAM; Profesora-Investigadora en el Departamento de Economía de la UAM-Iztapalapa, Coordinación de Administración, Área Planeación Estratégica de las Empresas. Correo electrónico: rmortegao@hotmail.com

${ }^{2}$ Doctor en Administración (Organizaciones), FCA-UNAM; Profesor-Investigador en el Departamento de Economía de la UAM-Iztapalapa, Coordinación de Administración, Área Planeación Estratégica de las Empresas, y de la FCA de la UNAM; Miembro del SNI. Correo electrónico: racornejo@hotmail.com

${ }^{3}$ Doctor en Administración (Organizaciones), FCA-UNAM; Profesor-Investigador en el Departamento de Economía de la UAM-Iztapalapa, Coordinación de Administración, Área Planeación Estratégica de las Empresas, y de la FCA-UNAM. Correo electrónico: evillegash@hotmail.com
} 
permitirá, con base en los resultados históricos, maximizar el capital que se aporta en las acciones de las Siefores, habida cuenta de que la inversión en Bolsa constituye una estrategia de largo plazo, justamente lo que realizan las Siefores.

Palabras clave: inversiones, BMV, Afores, Siefores, rendimiento. Clasificación JEL: G11, G23, N26.

\section{ABSTRACT}

In this work we seek to account for the importance of investments, considering that the purpose of any investor is to maximize their value. The issue is relevant for all those who invest in the Stock Exchange and especially for the pensions of the elderly, an issue that crosses the attention of governments, private initiative, workers and non-profit organizations, since it may be the only way to avoid a future population of elderly people living in poverty. The aim is to ensure an adequate flow of income when it is suspended due to old age, disability or work risks. Therefore, the investment that workers make through the Siefores must be properly managed. In this regard, it is important to see if the investment in the Stock Exchange will allow, based on the historical results, to maximize the capital that is contributed in the shares of the Siefores, given that the investment in the Stock Exchange constitutes a long-term strategy, precisely what perform the Siefores.

Key words: investments, Stock Exchange, Afores, Siefores, performance. Clasificación JEL: G11, G23, N26.

\section{Introducción}

I propósito de cualquier inversionista es maximizar el valor de su inversión. Al invertir en acciones de empresas que cotizan en la Bolsa Mexicana de Valores (BMV) lo obtienen cuando la empresa logra su objetivo financiero de maximizar su valor, medido esto a través del precio de sus acciones. Uno de los principales problemas que enfrentan los inversionistas es cómo medir el valor de sus inversiones en la BMV. Esta medición la pueden hacer en pesos corrientes afectados por la inflación, en pesos constantes, en dólares estadounidenses, o tal vez en euros. 
Para varios autores y algunos académicos del campo de las finanzas, el conocimiento financiero se da junto con la evolución del sistema bancario; por ende, nos remontaremos al siglo $\mathrm{VII}$ antes de Cristo, donde se dieron la primera letra de cambio, el primer certificado bancario y las primeras órdenes de pago.

Para Van Horne, fue en el siglo XX cuando las finanzas se separan del estudio de la economía y emergen como un campo de conocimiento autónomo. El mismo Van Horne señala que con la innovación tecnológica y las nuevas industrias, en la década de los años veinte, las empresas requirieron de recursos financieros, centrándose el énfasis en la liquidez y el financiamiento. Hacia el final de esa década, con lo que el papel del banquero inversionista predominó.

En los años setenta del siglo pasada se dio el modelo de valuación de activos que por sus siglas en inglés se conoce como CAPM, de Sharpe y en 1973 Black y Scholes regresan el énfasis al área económica con su modelo para valuar opciones. En los años ochenta y noventa del mismo siglo continuó la importancia del enfoque económico y Rappaport, al hablar sobre la creación de valor para el accionista, dio un enfoque diferente al que se tenía al partir de los estados financieros (Rappaport, 1986). Este enfoque se fortaleció en 1991 con el modelo EVA (Economic Value Added) (Stewart, 1991), que se refiere a la búsqueda del valor.

Un señalamiento fundamental de Van Horne es aquel que dice que "el objetivo de la empresa es maximizar la riqueza para sus accionistas. La riqueza está representada por el precio de mercado de las acciones comunes de la empresa" (Van Horne, 1977).

La importancia del tema se justifica en el hecho que México vive actualmente un momento en el que la edad promedio de sus habitantes está siendo modificada. En la década de los cincuenta se vivió un importante crecimiento de la población; ésta ha llegado a su madurez y pronto llegará a edad avanzada. Por otro lado, las familias cada vez tienen menos hijos; así, en algunos años, la población tendrá más adultos que niños. Aunado a lo anterior, a partir de los años cincuenta la higiene y la seguridad social en México y los avances en la medicina lograron aumentar la esperanza de vida, por lo que ahora vivimos hasta edad muy avanzada.

Este cambio en la estructura poblacional deberá hacer que la sociedad busque un cambio cultural que redefina el papel de la senectud para lograr su participación activa en su comunidad y la sana convivencia entre las generaciones.

Las pensiones de los adultos mayores son un tema cada vez más importante que requiere ser atendido por todos los sectores del país: gobiernos, iniciativa 
privada, trabajadores y organizaciones no lucrativas, ya que será la única forma en que dejemos de tener ancianos pobres y descuidados.

Según la Organización Internacional del Trabajo (OIT), la seguridad social involucra el acceso al cuidado de la salud y la seguridad en los ingresos, particularmente en los casos de vejez, invalidez, riesgo de trabajo, maternidad o pérdida del empleo.

Los sistemas de pensiones buscan garantizar el flujo de los ingresos cuando éstos se suspenden por vejez, invalidez o riesgos de trabajo, y por ende debe administrarse adecuadamente la inversión que las personas realizan en la Siefores para lograr esta meta.

Uno de los caminos para lograr esto es maximizar el valor de la inversión que las Sociedades de Inversión Especializadas e Fondos para el Retiro (Siefores) orientan hacia la BMV. Por tanto, la pregunta que dirige este trabajo es la siguiente: ¿Es alcanzable el objetivo financiero de maximizar el valor a través de la inversión en la BMV para el capital de retiro de los trabajadores?

Las hipótesis a verificar son que la inversión en Bolsa no logra maximizar el valor de las aportaciones de los trabajadores para maximizar su capital de retiro, y que la inversión en Bolsa si logra maximizar el valor de las aportaciones de los trabajadores para maximizar su capital de retiro.

El objetivo de la investigación es estimar si el invertir en la Bolsa permitirá, con base en los resultados históricos, maximizar el capital que se aporta en las acciones de las Siefores. La cultura financiera nos enseña que la inversión en la Bolsa es una idecisión de largo plazo y esto es consistente con lo que hacen las Siefores.

\section{Siefores}

Las sociedades de inversión, administradas y operadas por las administradoras, tienen por objeto invertir los recursos provenientes de las cuentas individuales que reciban en los términos de las leyes de seguridad social. Las administradoras son entidades financieras que se dedican de manera habitual y profesional a administrar las cuentas individuales y canalizar los recursos de las subcuentas que las integran en términos de la presente ley. 


\section{Régimen de inversión de las Siefores}

El régimen de inversión deberá tener como principal objetivo otorgar la mayor seguridad y rentabilidad de los recursos de los trabajadores. Asimismo, el régimen de inversión tenderá a incrementar el ahorro interno y el desarrollo de un mercado de instrumentos de largo plazo acorde con el sistema de pensiones. A tal efecto, proveerá que las inversiones se canalicen preponderantemente, a través de su colocación en valores, a fomentar:

- La actividad productiva nacional;

- La mayor generación de empleo;

- La construcción de vivienda;

- El desarrollo de infraestructura estratégica del país, y

- El desarrollo regional.

Las sociedades de inversión deberán operar con valores, documentos, efectivo y los demás instrumentos que se establezcan en el régimen de inversión que mediante reglas de carácter general establezca la Comisión, oyendo previamente la opinión del Banco de México, de la Comisión Nacional Bancaria y de Valores y del Comité Consultivo y de Vigilancia.

CUADRO 1. INFORME DE LAS CLASIFICACIONES MORNINGSTAR 2019 PARA LAS SIEFORE

\begin{tabular}{lc}
\hline \multicolumn{2}{c}{ Clasificación global } \\
\cline { 1 - 1 } SURA & PLATA \\
\hline CITIBANAMEX & BRONCE \\
\cline { 1 - 1 } PROFUTURO & NEUTRAL \\
\hline XXI BANORTE & \\
\hline AZTECA & NEGATIVO \\
\hline PRINCIPAL & \\
\hline COPPEL & \\
\hline INBURSA & \\
\hline INVERCAP & \\
\hline PENSIONISSSTE &
\end{tabular}

Los cinco pilares evaluados por Morningstar son: 
a. Proceso. - se identifica que la estrategia de inversión sea congruente con el objetivo final de los recursos;

b. Rendimiento. - se evalúa si el fondo se desempeña apegado a los objetivos de su estrategia de inversión, obteniendo en un periodo relevante un nivel adecuado de rendimiento ajustado por riesgo;

c. Personas. - implica el contraste de los objetivos de la estrategia de inversión, clases de activo y tipos de operaciones en las que se invierte, frente a las capacidades profesionales y experiencia de los equipos de inversión que ejecuta dicha estrategia;

d. Precio. - evaluación de la comisión del fondo en función del tipo de estrategia de inversión y su complejidad, proceso de inversión y calificaciones profesionales del equipo de inversión.

e. El desarrollo de infraestructura estratégica del país, y

f. Administradora. - se identifica donde se ubican las prioridades del gestor de fondos. En este sentido los analistas favorecen a aquellos gestores que priorizan el proceso de inversión sobre la promoción comercial.

CUADRO 2. CLASIFICACIONES MORNINGSTAR

\begin{tabular}{lccc}
\hline \multicolumn{4}{c}{ Evolución de las clasificaciones } \\
\hline Afore & $\mathbf{2 0 1 7}$ raiting & $\mathbf{2 0 1 8}$ raiting & $\mathbf{2 0 1 9}$ raiting \\
\hline SURA & BRONCE & PLATA & PLATA \\
\hline PROFUTURO & BRONCE & BRONCE & BRONCE \\
\hline CITIBANAMEX & BRONCE & BRONCE & BRONCE \\
\hline XXI BANORTE & NEUTRAL & NEUTRAL & BRONCE \\
\hline PRINCIPAL & NEUTRAL & NEUTRAL & NEUTRAL \\
\hline AZTECA & NEGATIVO & NEUTRAL & NEUTRAL \\
\hline INVERCAP & NEUTRAL & NEUTRAL & NEGATIVO \\
\hline COPPEL & NEGATIVO & NEGATIVO & NEGATIVO \\
\hline PENSIONISSSTE & NEUTRAL & NEGATIVO & NEGATIVO \\
\hline INBURSA & NEUTRAL & NEGATIVO & NEGATIVO \\
\hline
\end{tabular}

Con base en las calificaciones que la SIEFORE obtiene en cada uno de los pilares se les califica en una escala que va de negativo a oro.

a. Oro. - se asigna a los fondos que se destacan en los cinco pilares evaluados en el proceso; 
b. Plata. - se otorga a aquellos fondos cuyas capacidades sobresalen a lo largo de los cinco pilares, frente a las áreas de oportunidad identificadas;

c. Bronce. - son fondos con ventajas notables en varios de los cinco pilares (no en todos). Sin embargo, presentan áreas de oportunidad en algunas de las categorías evaluadas;

d. Neutral. - fondos cuyas capacidades y proceso no garantizan un desempeño superior al resto de la oferta en el mercado, y por lo tanto los analistas de Morningstar no consideran que puedan superar el desempeño del resto de la oferta en el mercado, $y$

e. Negativa. - fondos que de acuerdo con los analistas de Morningstar, presentan desventajas que inhibirán un buen desempeño futuro en comparación al resto de la oferta en el mercado.

CuAdro 3. EVolución dE LAS CLASIFICACIONES

\begin{tabular}{|c|c|c|c|c|c|c|}
\hline \multicolumn{7}{|c|}{ Clasiicaciones analista MORNINGSTAR } \\
\hline \multicolumn{2}{|c|}{ Clasificación global 2019} & Proceso & Rendimiento & Personas & Administradora & Precio \\
\hline SURA & PLATA & Positivo & Positivo & Positivo & Positivo & Neutral \\
\hline CITIBANAMEX & \multirow{3}{*}{ BRONCE } & Positivo & Positivo & Positivo & Positivo & Neutral \\
\hline PROFUTURO & & Positivo & Positivo & Positivo & Positivo & Neutral \\
\hline XXI BANORTE & & Positivo & Neutral & Positivo & Neutral & Neutral \\
\hline AZTECA & \multirow{2}{*}{ NEUTRAL } & Negativo & Neutral & Neutral & Neutral & Negativo \\
\hline PRINCIPAL & & Neutral & Negativo & Neutral & Neutral & Negativo \\
\hline COPPEL & \multirow{4}{*}{ NEGATIVO } & Negativo & Neutral & Negativo & Neutral & Negativo \\
\hline INBURSA & & Negativo & Negativo & Negativo & Neutral & Neutral \\
\hline INVERCAP & & Negativo & Negativo & Neutral & Neutral & Negativo \\
\hline PENSIONISSSTE & & Negativo & Neutral & Negativo & Negativo & Positivo \\
\hline
\end{tabular}

Los límites de inversión por tipo de activo son:

a. SBO. - La Sociedad de Inversión Básica o es para personas mayores de 60 años próximos a la jubilación y deberá invertir, sus recursos en títulos de deuda nacional;

b. SB1. - La Sociedad de Inversión Básica 1 deberá invertir los recursos de trabajadores que tengan 60 años de edad o más que no les corresponda invertir sus recursos en la Sociedad de Inversión Básica de Pensiones y los recursos de los pensionados bajo la modalidad de retiros programados; 
c. SB2. - La Sociedad de Inversión Básica 2 deberá invertir los recursos de Trabajadores que tengan entre 46 y 59 años de edad;

d. SB3. - La Sociedad de Inversión Básica 3 deberá invertir los recursos de Trabajadores que tengan entre 37 y 45 años de edad, y

e. SB4. - La Sociedad de Inversión Básica 4 deberá invertir los recursos de Trabajadores que tengan 36 o menos años de edad.

El que puedan invertir las SB2, 3 y 4 hasta el $45 \%$ en valores de renta variable justifica la importancia a la pregunta planteada al inicio de este trabajo.

CUADRO 4. LÍMITES DE INVERSIÓN POR CLASE DE ACTIVO

\begin{tabular}{cccccc}
\hline \multicolumn{5}{c}{ Límites de inversión por clase activo } \\
\hline & SB0 & SB1 & SB2 & SB3 & SB4 \\
\hline Renta variable & $0 \%$ & $10 \%$ & $30 \%$ & $35 \%$ & $45 \%$ \\
\hline
\end{tabular}

Incluye acciones individuales, IPOs, índices accionarios domésticos e intrnscionales, incluidos en la Relación de Índices, y obligaciones forzosamente convertibles en acciones de emisoras nacionales.

Fuente: http://www.consar.gob.mx/gobmx/Aplicativo/Limites_Inversión/ 3 de noviembre de 2019

\section{3. Índices de la BMV}

Para valorar si la inversión en la Bolsa Mexicana de Valores Sociedad Anónima de Capital Variable (S.A.B. de C.V.) ha logrado maximizar el patrimonio de la inversión que pudieron realizarse con los recursos de las Siefores valoraremos de enero de 2011 a octubre de 2019 el comportamiento que tuvieron los índices principales en Pesos corrientes, en Pesos constantes, en dólares americanos y en Euros.

\section{CUADRO 5. ÍNDICES PRINCIPALES DE LA BMV}

\begin{tabular}{ll}
\hline S\&P/BMV IPC & S\&P/BMV HABITA RT \\
\hline S\&P/BMV INMEX RT & S\&P/BMV FIBRAS \\
\hline S\&P/BMV IRT CompMX & S\&P/BMV IPC SISTENTABLE \\
\hline S\&P/BMV IRT LargeCamp & S\&P/BMV BURSA ÓPTIMO \\
\hline S\&P/BMV IRT MidCamp & \\
\hline
\end{tabular}

Fuente: http://www.bmv.com.mx 
Los índices de la Bolsa Mexicana de Valores, S.A.B. y S\&P Dow Jones, son indicadores cuyo objetivo es reflejar el comportamiento del mercado accionario mexicano o de algunos grupos de empresas que tengan características en común. Son conocidos y se consideran como de rendimiento simple ya que en su cálculo se consideran las variaciones que tengan los precios de las acciones que conforman el respectivo índice.

\section{S\&P/BMV IPC}

Es el principal indicador de Mercado Mexicano de Valores; expresa el rendimiento del mercado accionario en función de las variaciones de precios de una muestra balanceada, ponderada y representativa del conjunto de Emisoras cotizadas en la Bolsa, basado en las mejores prácticas internacionales..... tiene como principal objetivo, constituirse como un indicador representativo del Mercado Mexicano para servir como referencia y subyacente de productos financieros. (S\&P DOW JONES Indices, 2019)

La utilidad de este índice es que sirve de base para medir el rendimiento de las acciones que tengan mayor liquidez y que sean representativas de las empresas más grandes que cotizan en la Bolsa Mexicana de Valores, que abarque el mercado bursátil mexicano.

En la gráfica 1 se observa que en cifras corrientes el crecimiento promedio anual se va reduciendo conforme se reduce el período analizado, baja de $29.90 \%$ promedio anual en 40 años hasta 1.33\% en el período de 2011 a octubre de 2019.

\section{GRÁFICA 1.S\&P/BMV EN CIFRAS CORRIENTES}

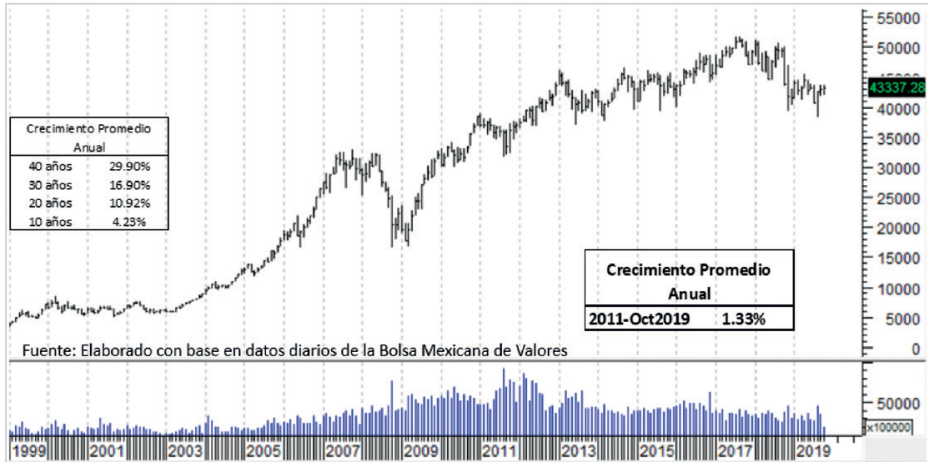


En la gráfica 2 se observa que en cifras constantes, el crecimiento promedio anual decrece de $7.18 \%$ en el período de 40 años hasta $-2.5 \%$ promedio anual en el lapso que va del año 2011 a octubre de 2019.

\section{GRÁFICA 2.S\&P/BMV EN CIFRAS CONSTANTES}

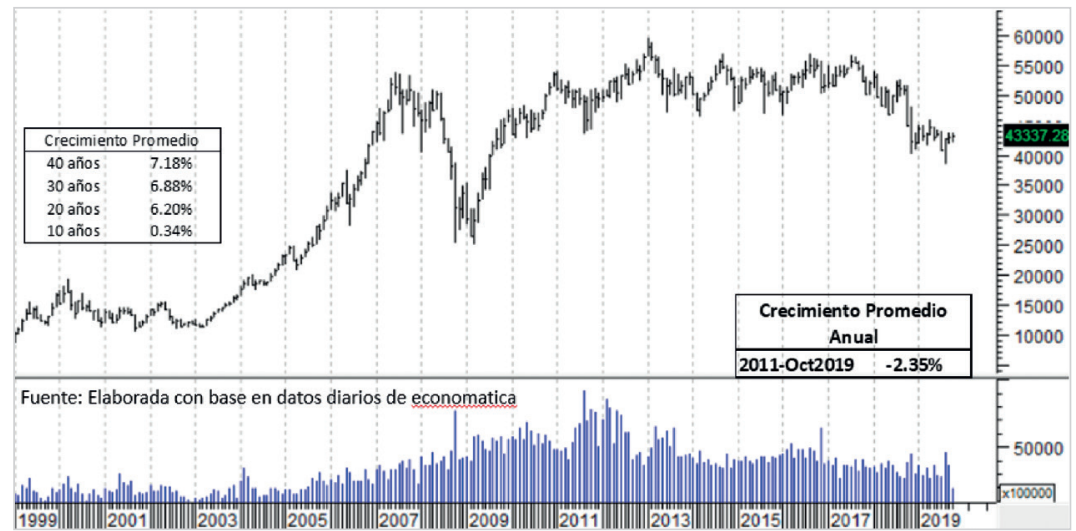

Medido el rendimiento en dólares americanos encontramos que los rendimientos bajan de $9.40 \%$ promedio anual en el lapso de 40 años hasta un decrecimiento en rendimiento de $\quad-3.38 \%$ promedio anual del año 2011 al mes de octubre de 2019.

\section{GRÁFICA 3.S\&P/BMV IPC EN USD}

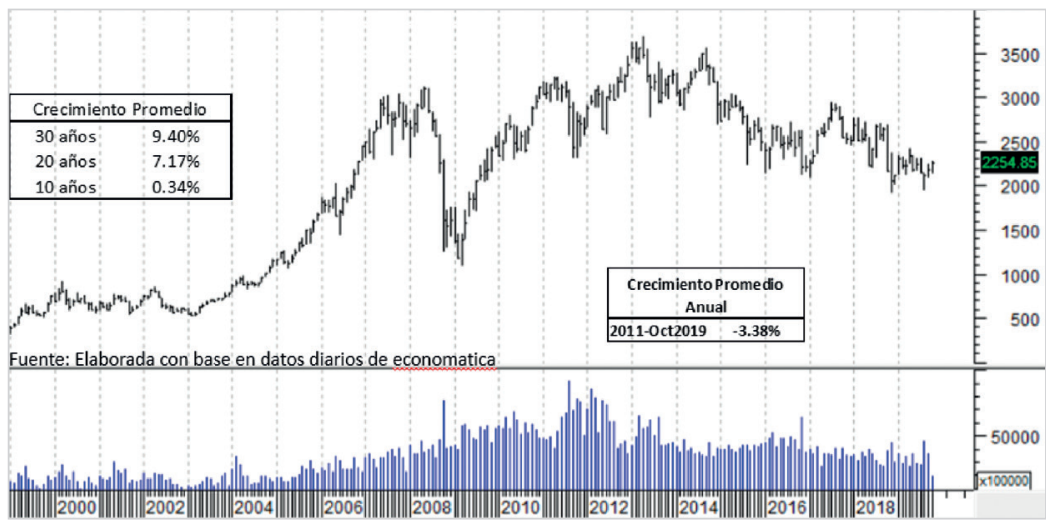


Como se observa en la gráfica 4, el comportamiento encontrado en cifras constantes y en términos de dólares americanos se replica parcialmente pues no se cuenta con datos en Euros de 40 años atrás, sin embargo sí se reduce el rendimiento promedio anual conforme se acorta el período analizado. Baja de 6.87\% promedio anual en 20 años a $-0.88 \%$ promedio anual del año 2011 al mes de octubre de 2019.

\section{GRÁFICA 4. S\&P/BMV IPC EN EUROS}

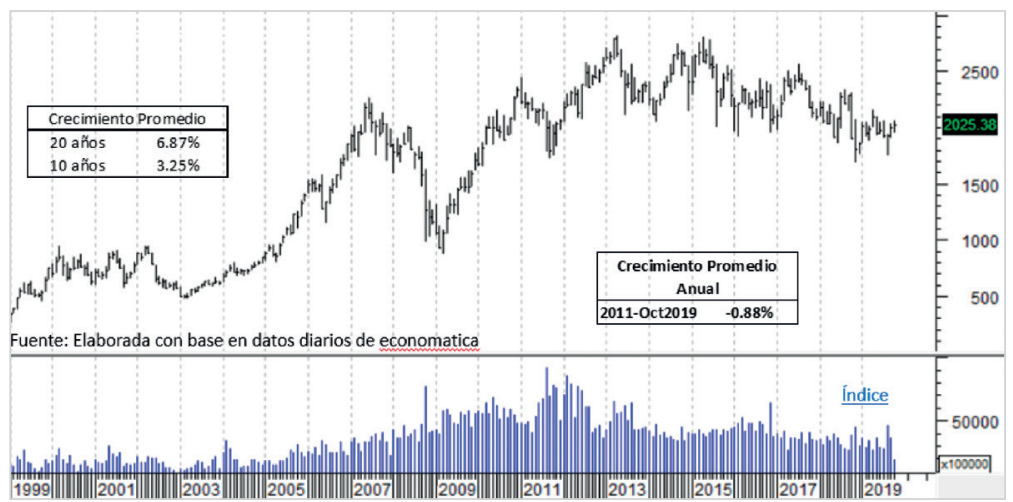

\section{S\&P/BMV INMEX RT}

La implementación de productos derivados en el mercado bursátil mexicano trajo consigo la necesidad de contar con un nuevo Índice de precios, el cual estuviera dentro de los estándares de cálculo y las políticas de mantenimiento de los Índices que existen en otros países y que tuviera además el reconocimiento en el ámbito internacional. El resultado es el S\&P/BMV Índice México (S\&P/BMV INMEX RT), un Índice de precios ponderado por valor de capitalización ajustado por acciones flotantes, el cual se constituye, al igual que el S\&P/BMV IPC, como un indicador altamente representativo y confiable del mercado accionario mexicano. Tiene la particularidad de tomar en cuenta para su rendimiento los dividendos en efectivo. (Bolsa Mexicana de Valores, S.A.B., 2019)

A través de la utilización de este índice se puede medir el rendimiento de las 20 acciones representativas de las empresas de mayor tamaño y liquidez en la operación del mercado bursátil mexicano, estás acciones también son consideradas en el S\&P BMV IPC. 


\section{GRÁFICA 5. S\&P/BMV INMEX EN CIFRAS CORRIENTES}

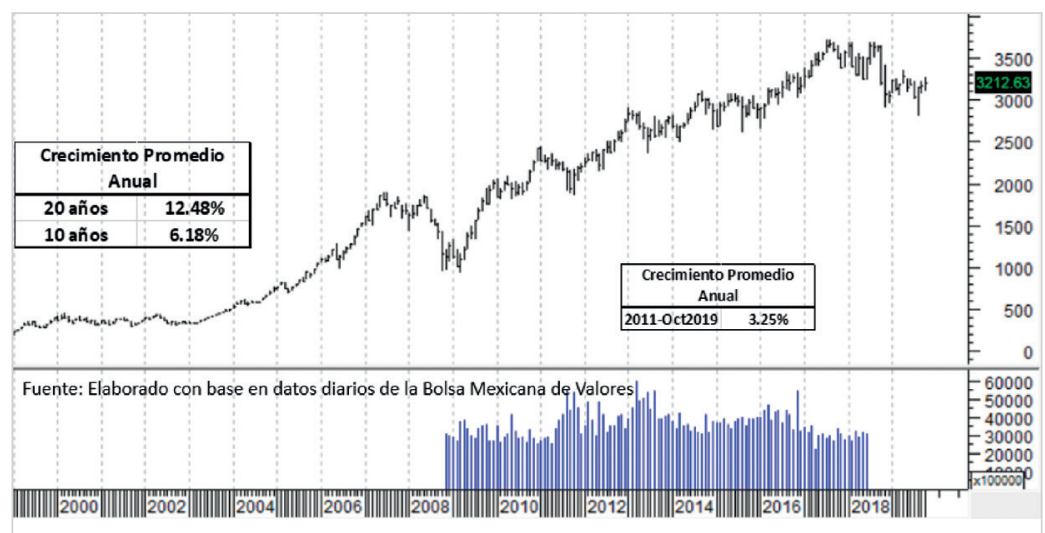

Este índice únicamente se pudo analizar en su comportamiento de 20 años y se encontró que conforme se acorta el tiempo de análisis su rendimiento se redujo de $12.48 \%$ promedio anual en un período de 20 años a $3.25 \%$ promedio anual en el período que abarca del año 2011 a octubre de 2019.

\section{GRÁFICA 6. S\&P/BMV INMEX EN CIFRAS CONSTANTES}

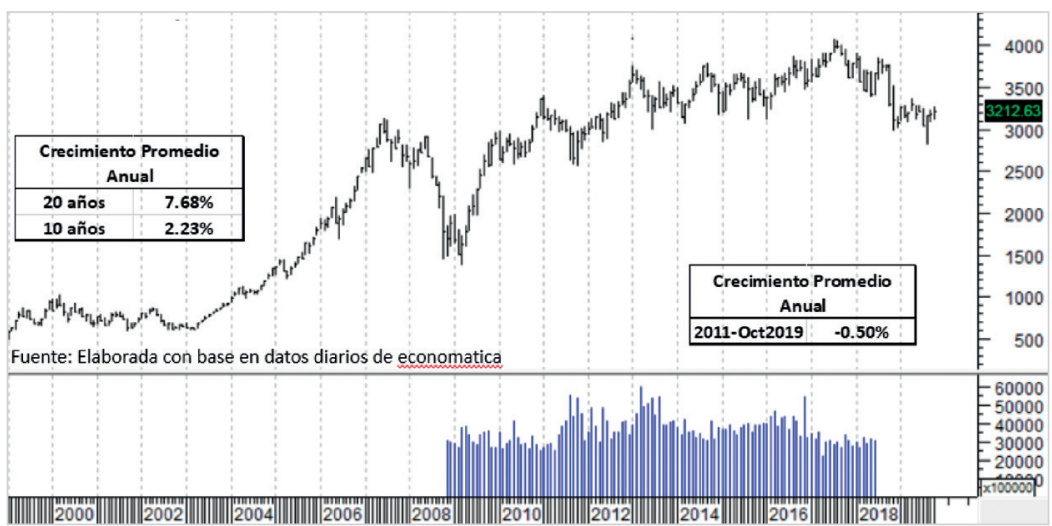

En cifras constantes el rendimiento redujo su crecimiento promedio anual de $7.68 \%$ en un lapso de 20 años a $-0.50 \%$ promedio anual en el período del año 2011 a octubre de 2019. 


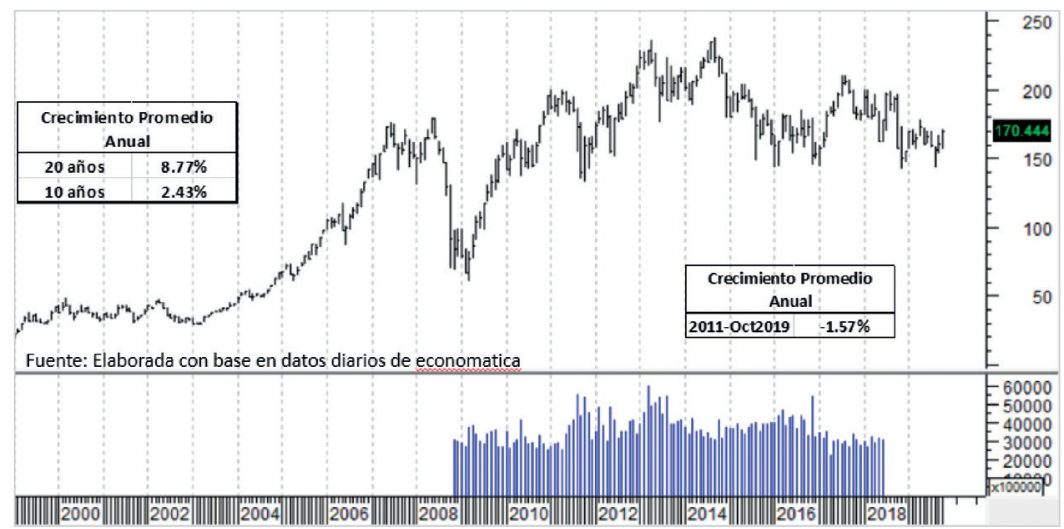

Medido en términos de dólares americanos, el rendimiento promedio anual se redujo, como se observa en la gráfica 7 de $8.77 \%$ promedio anual en 20 años a $-1.57 \%$ promedio anual de 2011 a octubre de 2019 .

\section{GRÁFICA 8. S\&P/BMV INMEX RT EN EUROS}

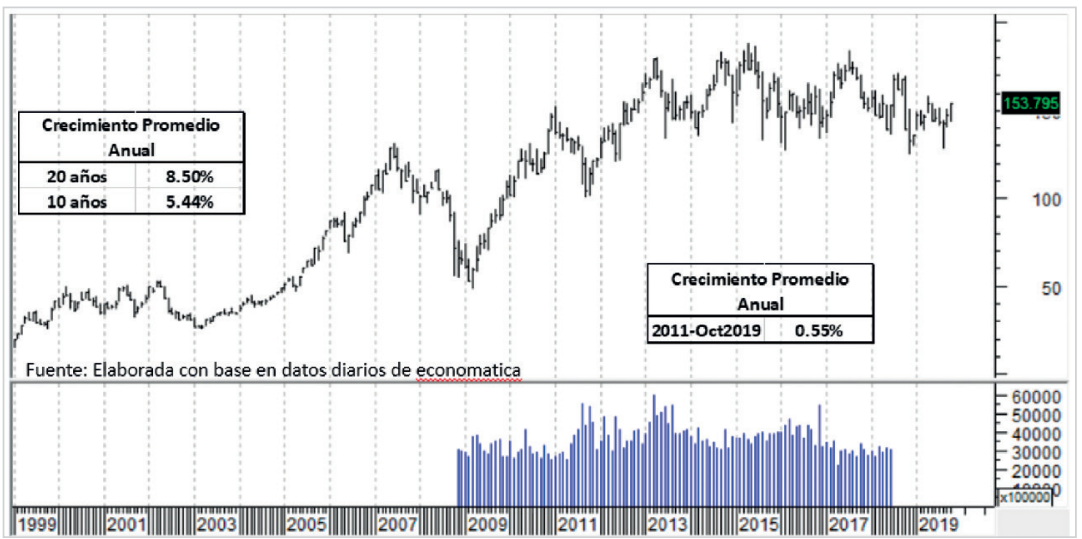

Medido en euros, el rendimiento promedio anual se redujo de $8.50 \%$ promedio anual en un lapso de 20 años a $0.55 \%$ promedio anual de 2011 a octubre de 2019, como se observa en la gráfica 8. 


\section{S\&P/BMV IRT CompMx}

El S\&P/BMV IRT CompMx es la variación de Rendimiento Total de su homónimo de Rendimiento Simple (S\&P/BMV IPC CompMx), esto es, que incorpora en su rendimiento todos los eventos corporativos, incluyendo los Dividendos en Efectivo.... refleja en forma amplia el comportamiento del mercado accionario mexicano al incluir en su muestra a las empresas más grandes y líquidas del mercado... considerando dos conceptos fundamentales:

- La representatividad de la muestra en cuanto a la dinámica operativa del mercado, la cual es asegurada mediante la selección de las emisoras líderes en operación y tamaño.

- La estructura de cálculo incorpora el valor de mercado de las emisoras ajustado por acciones flotantes, el cual actúa como ponderadory determina la contribución que cada una de las series accionarias tiene dentro de la muestra. (Bolsa Mexicana de Valores, S.A.B., 2019)

El índice S\&P/BMV IPC CompMx se utiliza como un parámetro de comparación amplio para el mercado bursátil mexicano. Busca medir el rendimiento de acciones de empresas mexicanas que cotizan en la Bolsa Mexicana de Valores con criterios mínimos de tamaño y liquidez y ponderados por capitalización de mercado además de estar sujetos a requisitos de diversificación.

Como se observa en la gráfica 9, su historia es menor y en promedio de los últimos 10 años generó un rendimiento promedio anual de $7.02 \%$, aún y cuando en el lapso del año 2011 a octubre de 2019 este rendimiento promedio se redujo a $4.09 \%$. 


\section{GRÁFICA 9. S\&P/BMV INMEX RT EN EUROS}

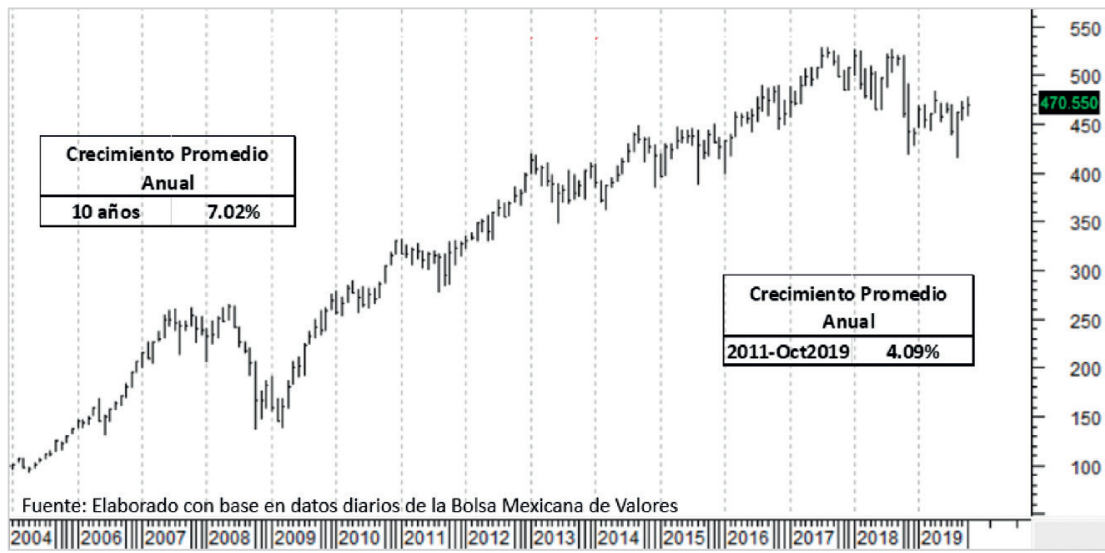

En cifras constantes el rendimiento promedio anual de los últimos 10 años fue de $3.03 \%$ y en el período del año 2011 a octubre de 2019 se redujo a $0.30 \%$ (Gráfica 10).

\section{GRÁFICA 10. S\&P/BMV IIRT COMPMX EN CIFRAS CONSTANTES}

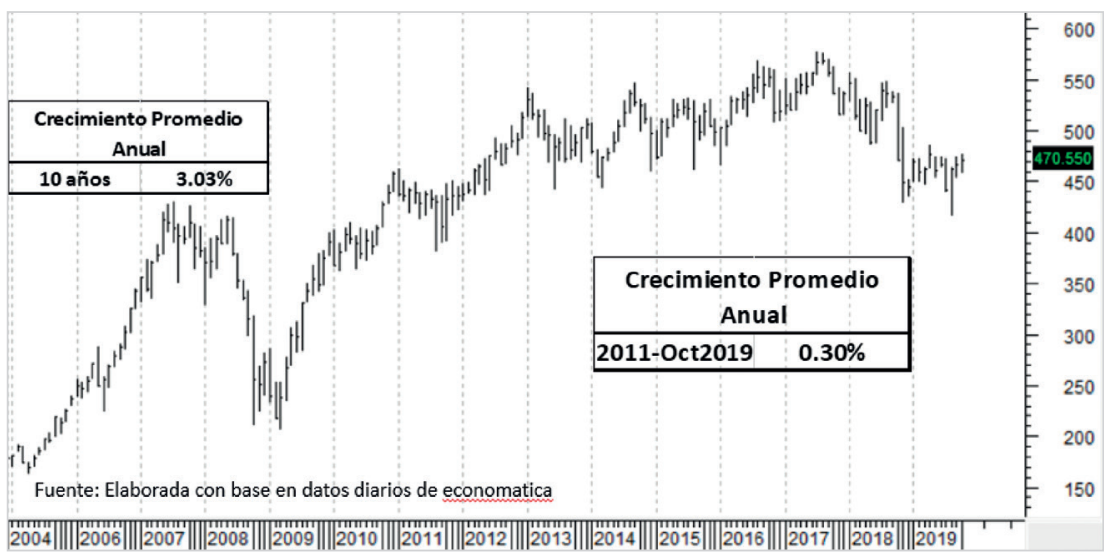

El rendimiento promedio anual en dólares americanos por los últimos 10 años fue de $7.17 \%$ y en el período que va del año 2011 a octubre de 2019 se redujo a $2.34 \%$, como se observa en la gráfica 11 . 


\section{GRÁFICA 11. S\&P/BMV IIRT COMPMX EN USD}

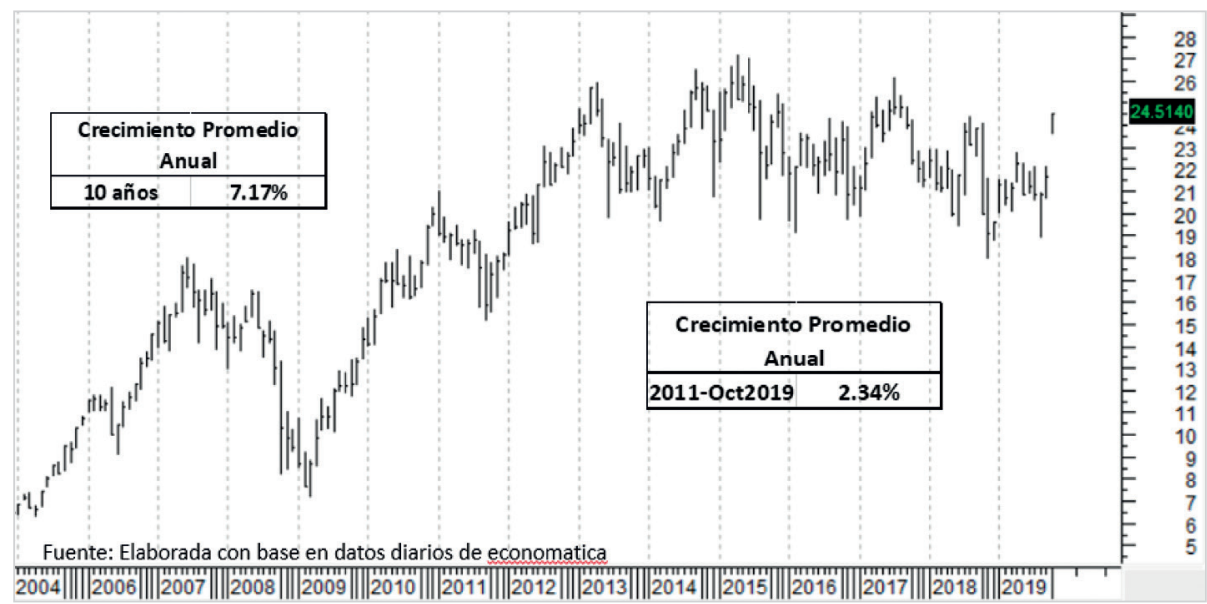

El rendimiento promedio anual en euros, en los últimos 10 años, fue de $6.01 \%$ y del año 2011 a octubre de 2019 se redujo a 1.09\%, como se observa en la gráfica 12.

\section{GRÁFICA 12.S\&P/BMV IIRT COMPMX EN EUROS}

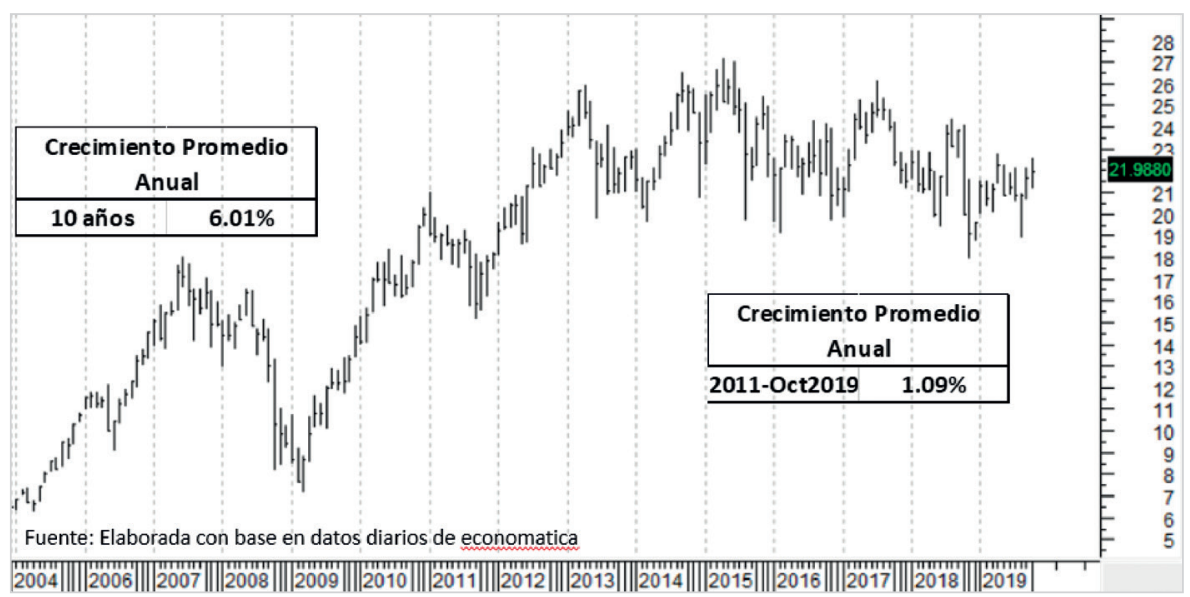




\section{S\&P/BMV IRT LargeCap}

El S\&P/BMV IRT LargeCap forma parte de la familia de Índices Compuestos de Rendimiento Total de la BMV: S\&P/BMV IRT CompMx, S\&P/BMV IRT LargeCap, S\&P/BMV IRT MidCap y S\&P/BMV IRT SmallCap. Es un indicador que refleja en forma amplia el comportamiento del mercado accionario mexicano al incluir en su muestra a las empresas más grandes dentro de la muestra. (Bolsa Mexicana de Valores, S.A.B., 2019)

\section{GRÁFICA 13. S\&P/BMV IRT COMPMX EN EUROS}

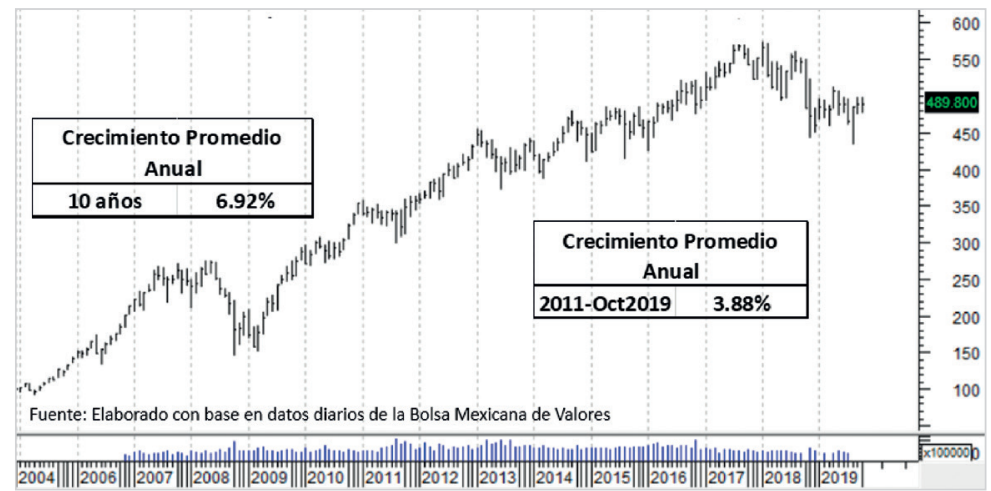

El rendimiento promedio anual en los últimos 10 años fue de $6.92 \%$ en tanto que del año 2011 a octubre de 2019 se redujo a 3.88\%.

\section{GRÁFICA 14. S\&P/BMV IRT LARGECAMP EN CIFRAS CONSTANTES}

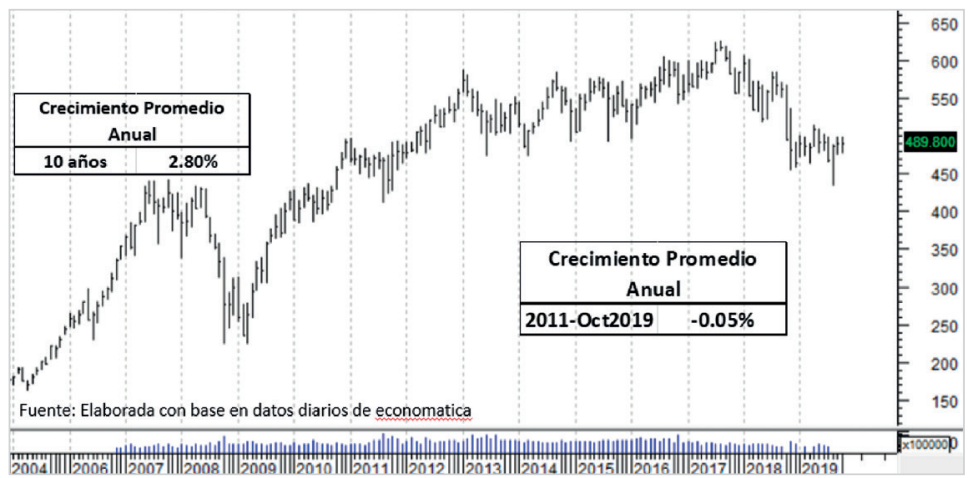


El rendimiento real promedio anual por los últimos 10 años, en cifras constantes, fue de $2.80 \%$, obteniéndose una cifra negativa promedio anual del año 2011 a octubre de 2019 de $-0.05 \%$.

\section{GRÁFICA 15. S\&P/BMV IRT LARGECAMP EN USD}

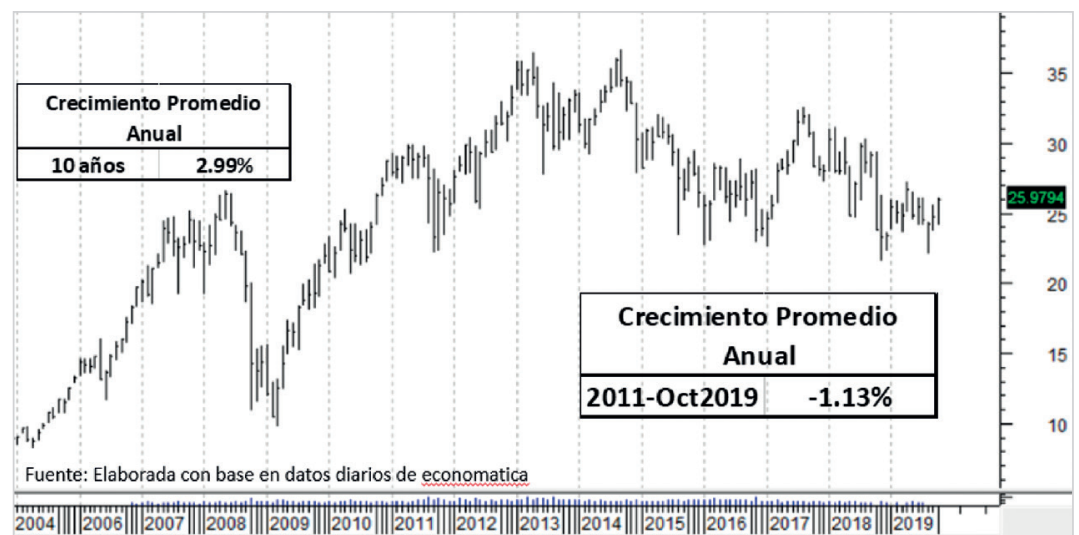

Medido en términos de dólares americanos, el crecimiento promedio anual por los últimos 10 años fue de $2.99 \%$ en tanto que del año 2011 a octubre de 2019 se redujo a $-1.13 \%$.

\section{GRÁFICA 16. S\&P/BMV IRT LARGECAMP EN USD}

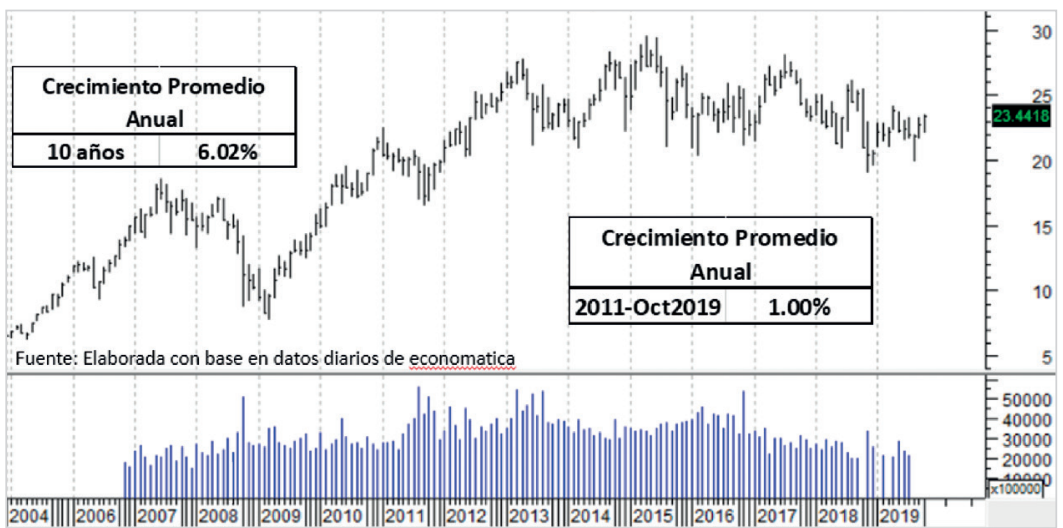


Medido en euros, el rendimiento promedio anual por los últimos 10 años fue de $6.02 \%$ y a partir del año 2011 y hasta octubre de 2019 se redujo a $1.00 \%$.

\section{S\&P/BMV IRT MidCap}

El Índice S\&P/BMV IPC MidCap es un indicador que refleja en forma amplia el comportamiento del mercado accionario mexicano al incluir en su muestra a las empresas de la parte media dentro de la muestra del Índice S\&P/BMV IRT CompMx. (Bolsa Mexicana de Valores, S.A.B., 2019)

Con el índice S\&P/BMV IPC MidCap se trata de medir el rendimiento de las acciones de capitalización media. Estas acciones representan aproximadamente el $20 \%$ de la capitalización del mercado, sin considerar las acciones de alta capitalización.

\section{GRÁFICA 17. S\&P/BMV IRT MIDCAMP EN CIFRAS CORRIENTES}

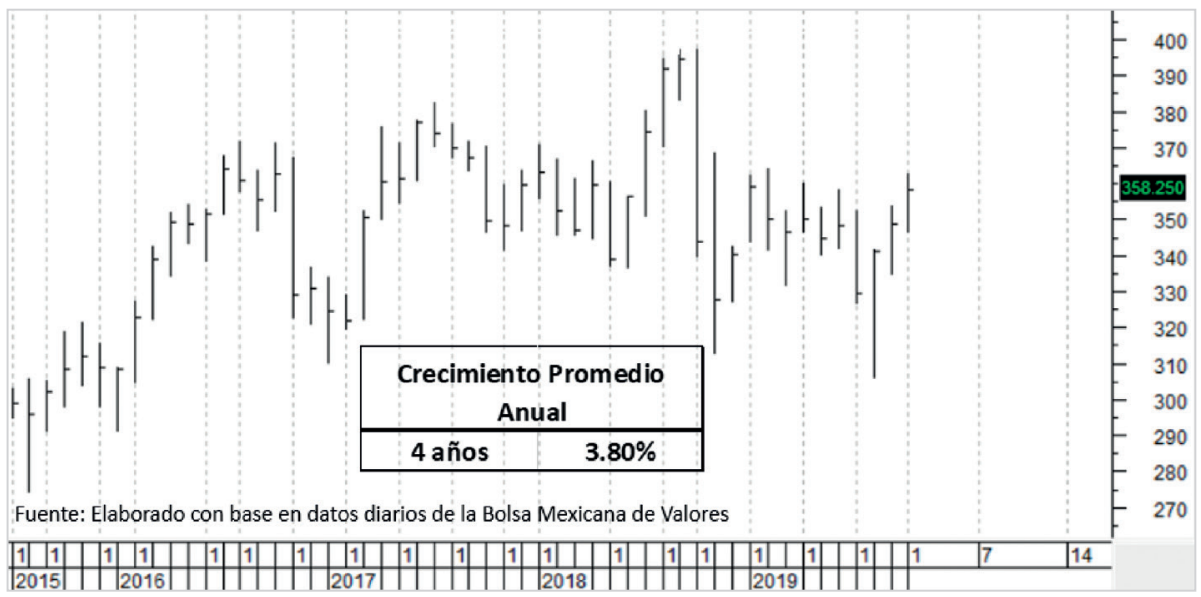

Como se observa en la gráfica 17, el rendimiento promedio anual en los último 4 años fue de $3.8 \%$. 


\section{GRÁFICA 18. S\&P/BMV IRT MIDCAMP EN CIFRAS CONSTANTES}

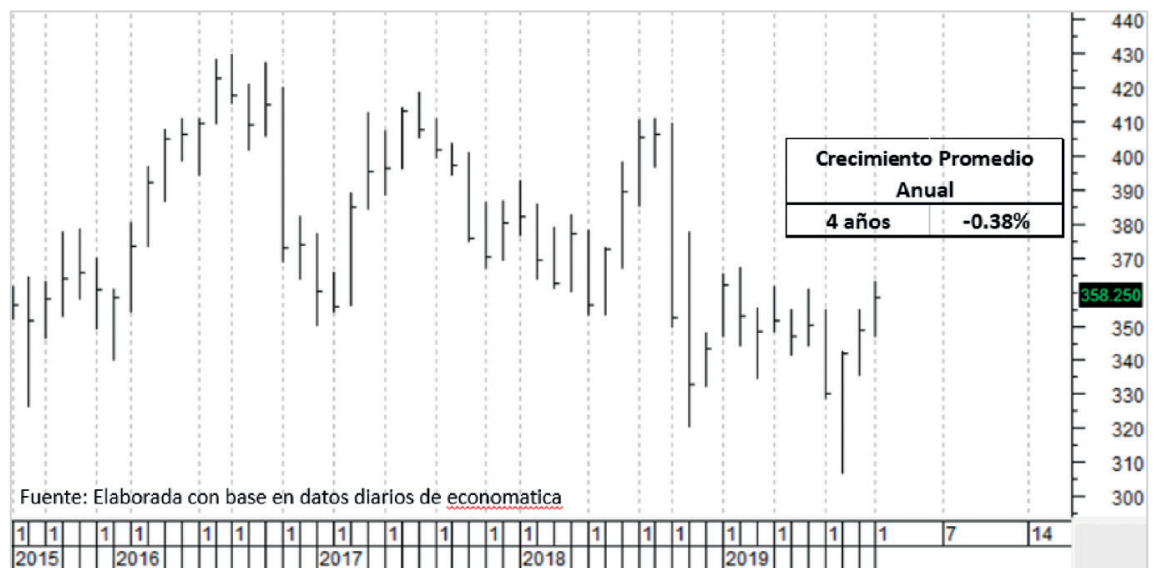

En cifras comparables, el rendimiento promedio anual fue de $-0.38 \%$, como se observa en la gráfica 18.

GRÁFICA 19. S\&P/BMV IRT MIDCAMP EN USD

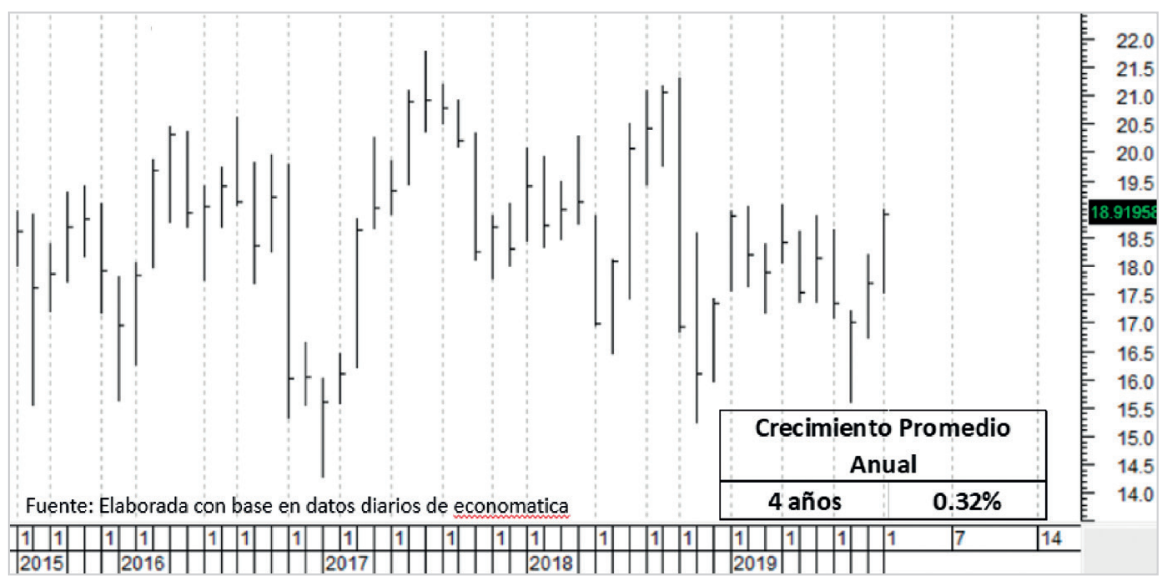

Analizando en términos de dólares americanos, el rendimiento promedio anual en los últimos 4 años fue de $0.32 \%$, como se observa en la gráfica 19. 


\section{GRÁFICA 20. S\&P/BMV IRT MIDCAMP EN USD}

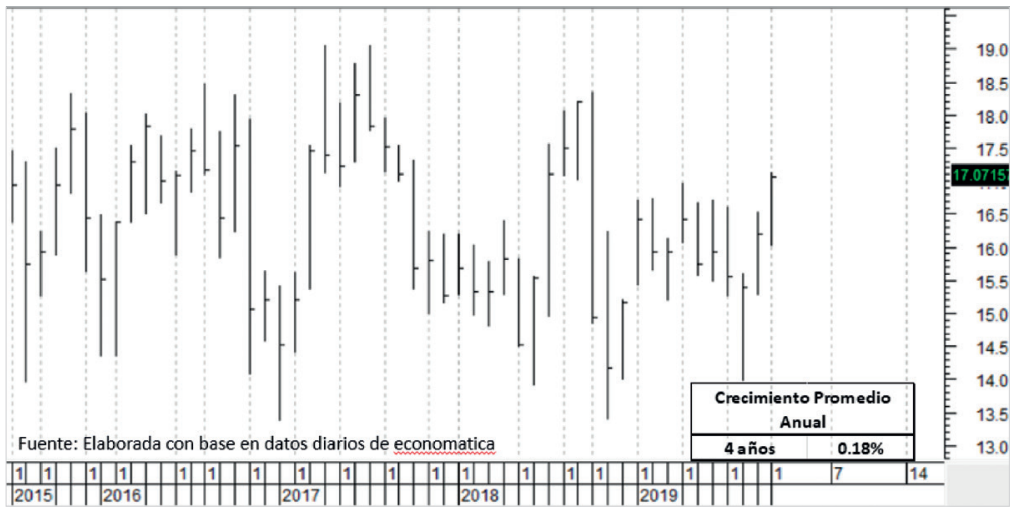

El rendimiento promedio anual en los últimos 4 años, en términos de Euros fue de tan solo $0.18 \%$, como se observa en la gráfica 20.

\section{S\&P/BMV HABITA RT}

La Bolsa Mexicana de Valores y S\&P desarrollaron un Índice dedicado al sector de la vivienda denominado Índice S\&P/BMV HABITA, este sector se ha constituido como un pilar muy importante en la economía del país en los últimos años. El Índice S\&P/BMV HABITA RT incorpora en su rendimiento todos los eventos corporativos, incluyendo los Dividendos en Efectivo. (Bolsa Mexicana de Valores, S.A.B., 2019)

\section{GRÁFICA 21. S\&P/BMV HABITA EN CIFRAS CORRIENTES}

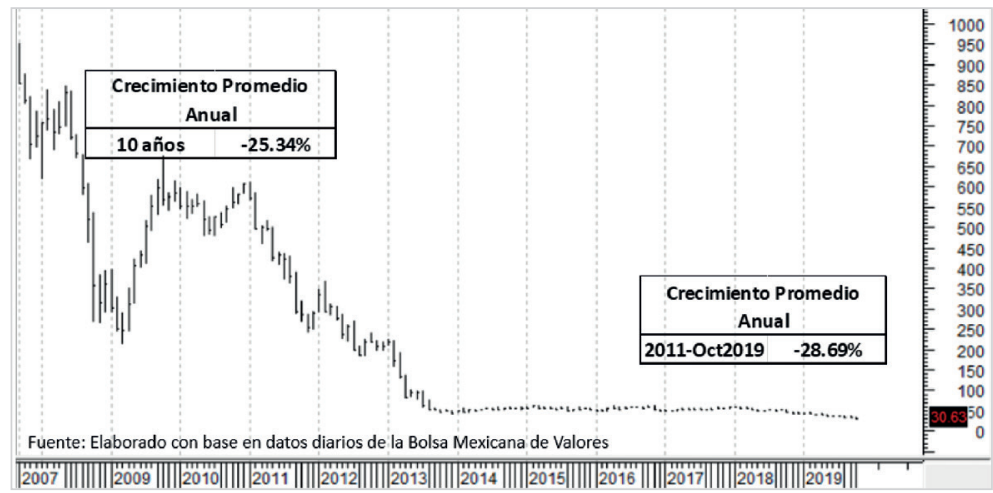


Es impresionante lo que este índice refleja del sector vivienda, en promedio, en cifras corrientes, durante los últimos 10 años perdió $25.34 \%$, y del año 2011 al mes de octubre de $201928.69 \%$, como se observa en la gráfica 21.

\section{GRÁFICA 22. S\&P/BMV HABITA EN CIFRAS CONSTANTES}

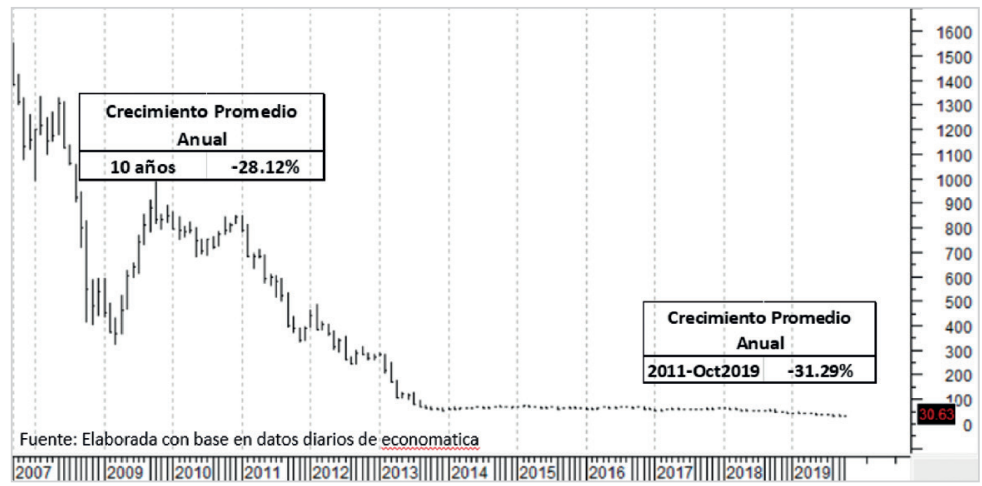

En cifras comparables, en la gráfica 22 se observa que perdió en promedio durante los últimos 10 años $28.12 \%$ y del año 2011 al mes de octubre de 2019,31.29\%.

\section{GRÁFICA 23. S\&P/BMV HABITA EN USD}

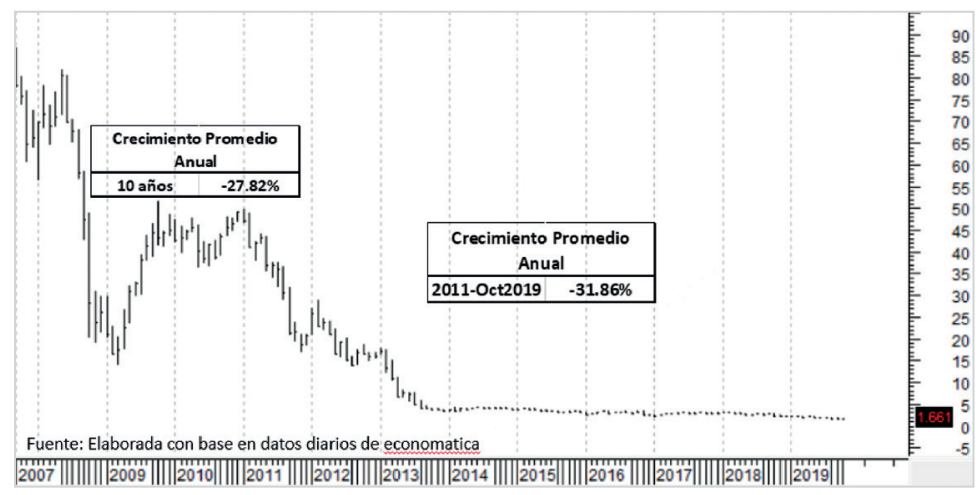

Las cifras en términos de dólares americanos son muy similares y como se observa en la gráfica 23, perdió durante los últimos 10 años, 27.82\% y del año 2011 al mes de octubre de $201931.86 \%$ (Gráfica 23). 
GRÁFICA 24. S\&P/BMV HABITA EN EUROS

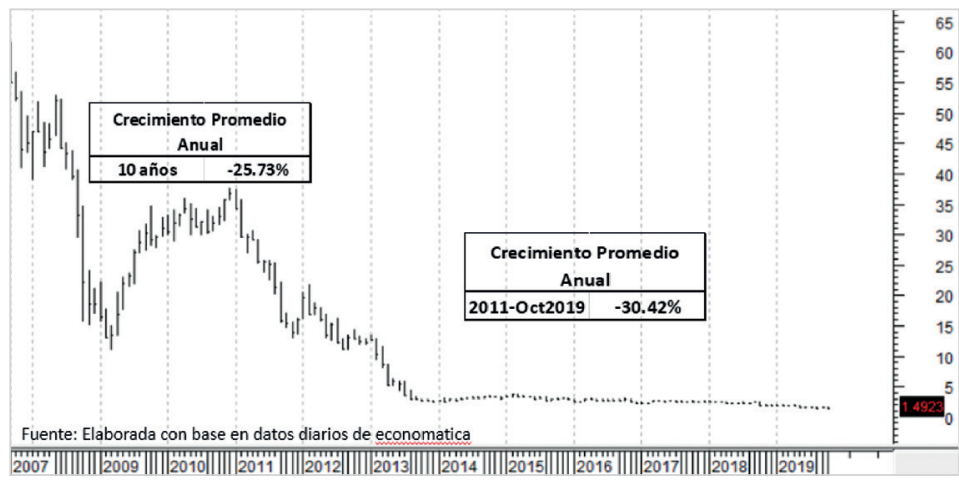

El comportamiento en euros resultó igual de malo, como se observa en la gráfica 24, en promedio perdió durante los últimos 10 años $25.73 \%$ y del año 2011 al mes de octubre de $201930.42 \%$.

\section{S\&P/BMV FIBRAS}

Índice dedicado a los Fideicomisos de Infraestructura y Bienes Raíces (FIBRAS), dicho Índice se ha denominado Índice S\&P/BMV FIBRAS. Estos vehículos de financiamiento de bienes raíces son una nueva clase de activos que son colocados en la Bolsa Mexicana de Valores, y el cual ha demostrado tener un comportamiento en su rendimiento distinto al del mercado accionario en lo general, considerando dos conceptos fundamentales:

Representatividad. La muestra que lo compone refleja el comportamiento y la dinámica operativa de esta clase de activos.

Invertibilidad. Los pesos relativos de la muestra se definen en función del importe operado en Bolsa, así se asegura que el Índice responde a las necesidades de operatividad y liquidez del mercado mexicano. (Bolsa Mexicana de Valores, S.A.B., 2019)

En términos más sencillos, las Fibras representan una alternativa de inversión equivalente a la inversión inmobiliaria con diferentes ventajas, entre otras las siguientes: 
- La liquidez, se puede realizar una compra o venta en cualquier momento.

- La valuación, muchas personas consideran la inversión en inmuebles como un buen negocio que cumple con el objetivo de maximizar su valor, el problema es que no se conoce cuánto vale un inmueble hasta que se trata de vender o comprar. En las Fibras se pueden consultar las cotizaciones todos los días conociéndose si se ha cumplido con el objetivo.

- El importe de inversión requerido, en un inmueble normalmente se requiere un desembolso elevado, en una Fibra no se compra un inmueble específico, se invierte en una cartera de inmuebles y el desembolso no es tan grande.

- Cuando una persona física compra un bien inmueble deseando hacer la mejor inversión, tal vez toma la decisión equivocada pues normalmente no es experto en la administración inmobiliaria. Las Fibras cuentan con administradores profesionales.

- Como en cualquier inversión, no existe la garantía de éxito ni en la inversión directa en inmuebles ni la inversión en Fibras, se requiere conocimiento para tratar de obtener los mejores resultados.

\section{GRÁFICA 25. S\&P/BMV HABITA EN CIFRAS CORRIENTES}

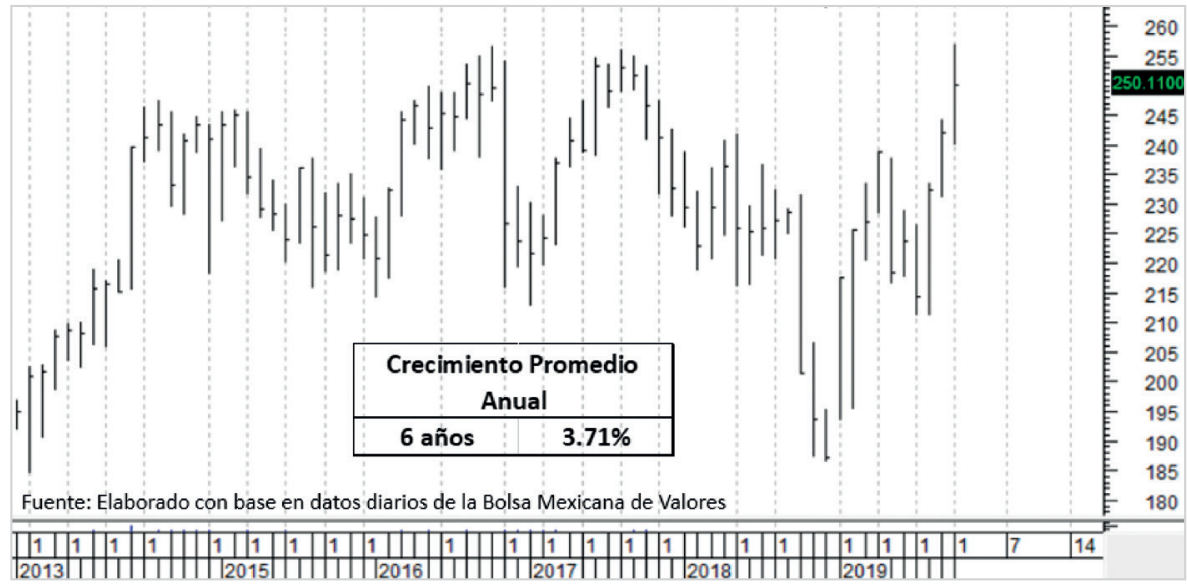

En cifras corrientes, se observa en la Gráfica 25 que el promedio de rendimiento del Índices S\&P/ BMV FIBRAS fue de 3.71\% promedio anual. 


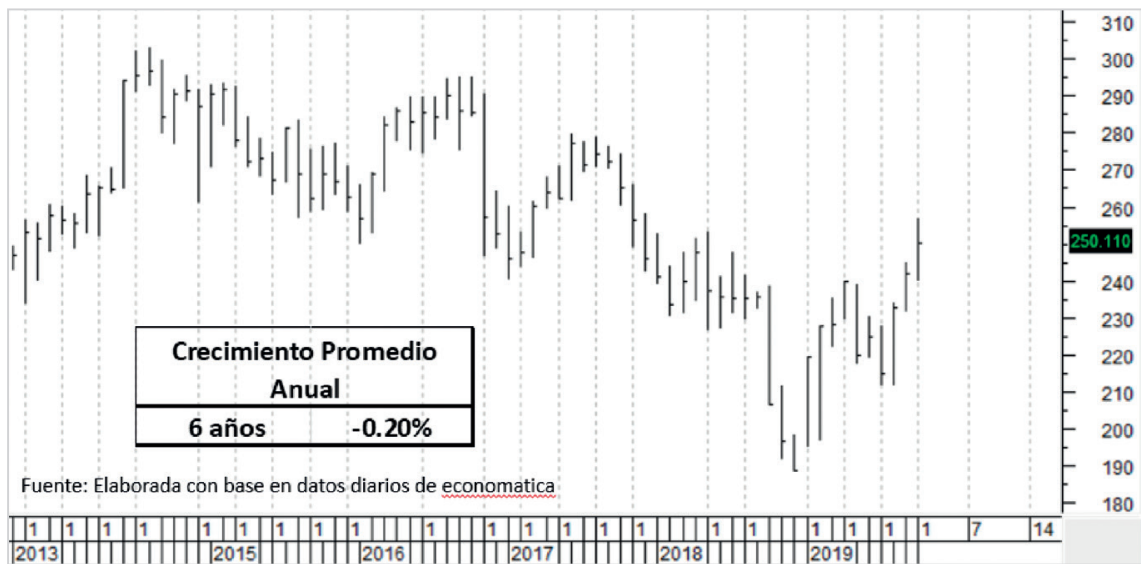

En la gráfica 26 se observa que en los últimos 6 años el rendimiento promedio anual de la inversión en Fibras fue de -0.20\%

\section{GRÁFICA 27. S\&P/BMV HABITA EN CIFRAS USD}

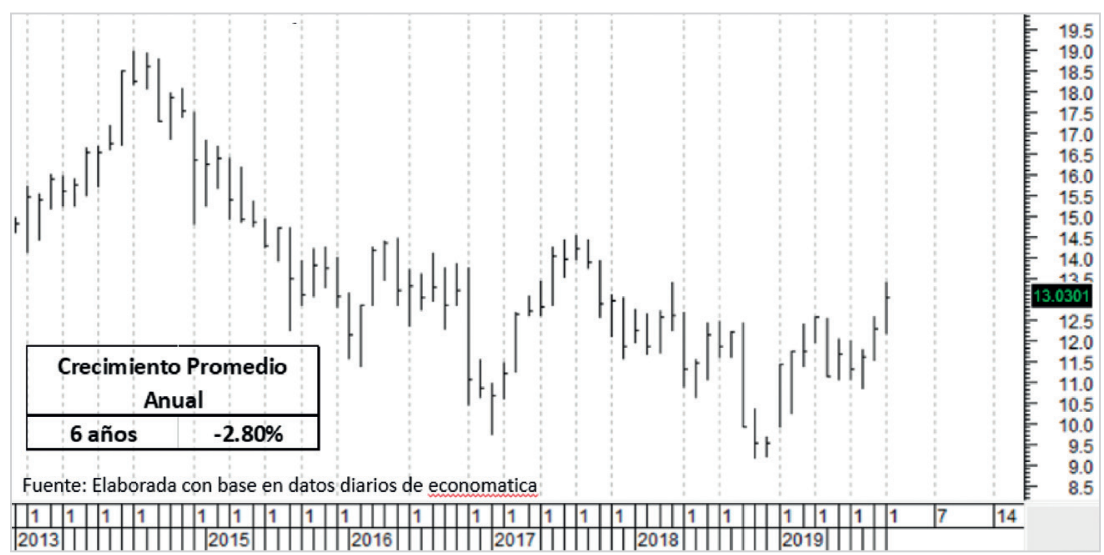

En términos de dólares americanos, en la gráfica 27 se observa que en los últimos 6 años el rendimiento promedio anual fue de $-2.80 \%$. 


\section{GRÁFICA 28. S\&P/BMV HABITA EN CIFRAS EUROS}

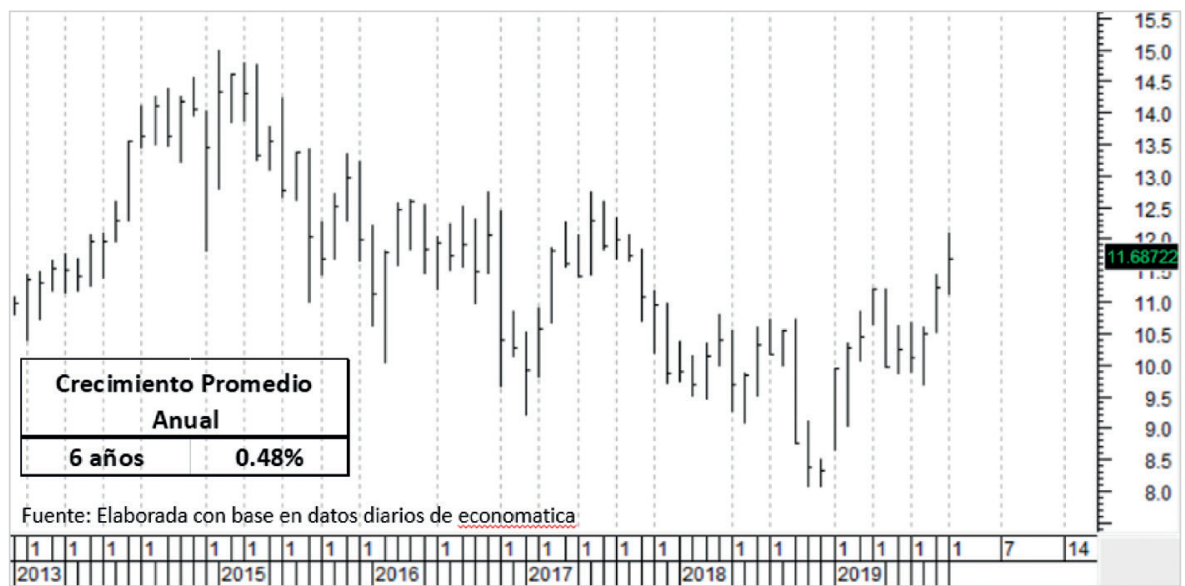

Si se hubiera considerado la inversión en Euros, el rendimiento promedio anual en 6 años hubiera sido de $0.48 \%$, como se muestra en la gráfica 28 .

\section{S\&P/BMV IPC SUSTENTABLE}

La Bolsa Mexicana de Valores, consciente de las tendencias internacionales en materia sustentable y de la creciente importancia que ha presentado en los mercados accionarios el tema ambiental, social y de gobierno corporativo, desde diciembre de 2011 decidió incorporar a su familia de índices un nuevo grupo de productos para el seguimiento del mercado accionario mexicano. (Bolsa Mexicana de Valores, S.A.B., 2019)

Algunos investigadores han considerado en sus propuestas de investigación como hipótesis que la inversión en acciones de empresas sostenibles debería brindar un mejor rendimiento que la inversión en otras acciones de empresas que no lo son. No lo han demostrado. 


\section{GRÁFICA 29. S\&P/BMV SUSTENTABLE EN CIFRAS CORRIENTES}

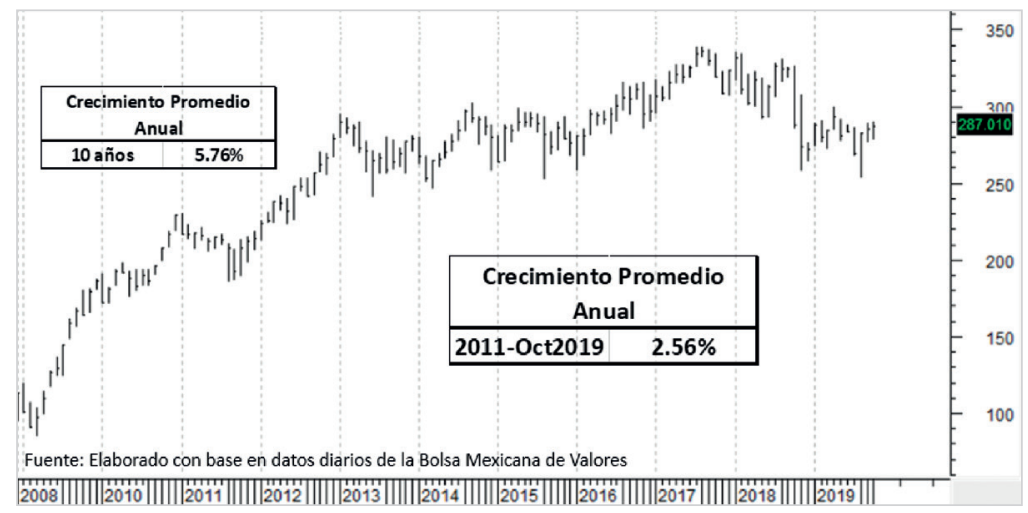

En la gráfica 29 se observa que el rendimiento promedio anual en 10 años ha sido de $5.76 \%$ y en el período que va del año 2011 al mes de octubre de 2019 de $2.56 \%$.

\section{GRÁFICA 30. S\&P/BMV SUSTENTABLE EN CIFRAS CONSTANTES}

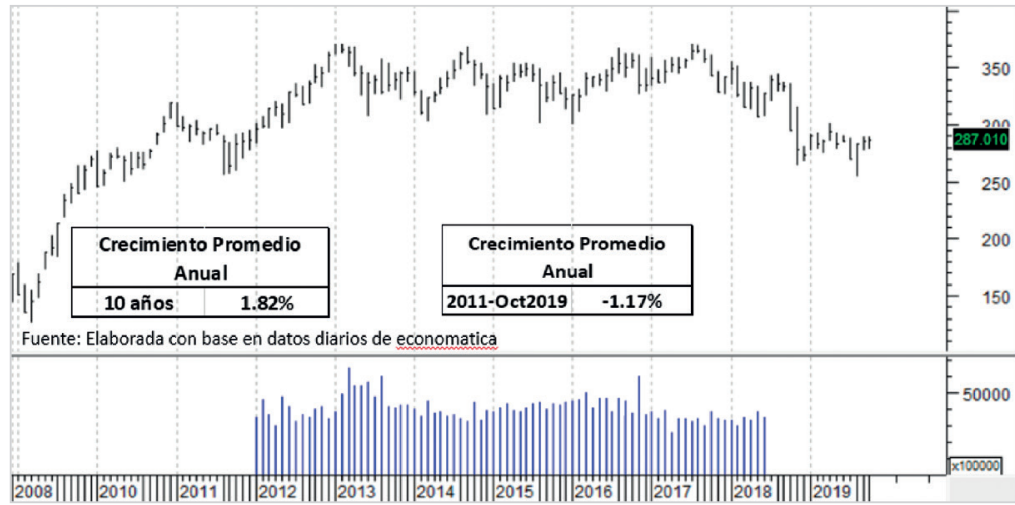

En términos reales, con cifras constantes se observa en la gráfica 30 que el rendimiento promedio anual en 10 años, invirtiendo en el índice sostenible (sustentable) fue de $1.82 \%$, sin embargo del año 2011 al mes de octubre de 2019 fue de $-1.17 \%$. 


\section{GRÁFICA 31. S\&P/BMV SUSTENTABLE EN USD}

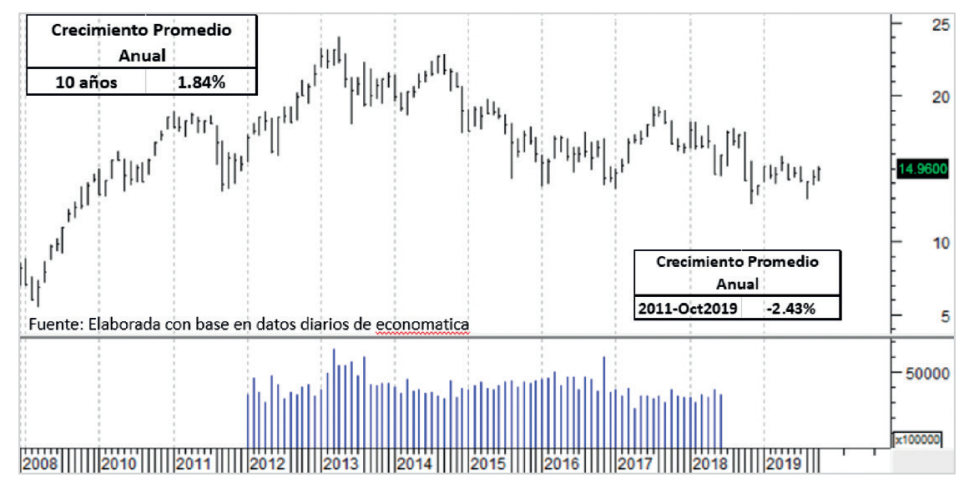

Si se hubiera considerado en términos de dólares americanos, la gráfica 31 muestra que el rendimiento promedio anual en los últimos 10 años habría sido de $1.84 \%$, pero en el período que va del año 2011 al mes de octubre de 2019 fue de $-2.43 \%$ promedio.

\section{GRÁFICA 32. S\&P/BMV SUSTENTABLE EN EUROS}

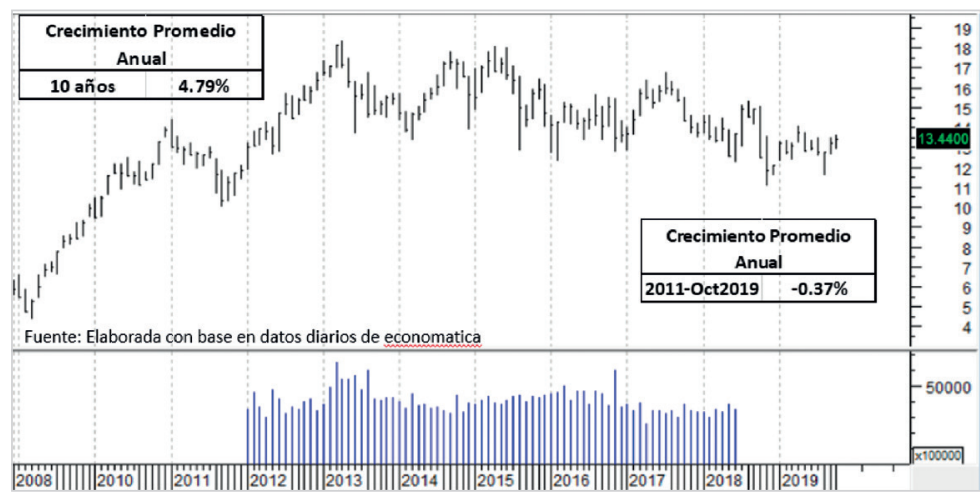

En caso de considerar el rendimiento en euros, la gráfica 32 muetra que en 10 años el rendimiento promedio anual hubiera sido de $4.79 \%$ pero si se hubiera invertido en el año 2011 y hasta el mes de octubre de 2019, el rendimiento promedio anual hubiera sido de $-0.37 \%$. 


\section{Índice S\&P/BMV BURSA ÓPTIMO}

Expresa el rendimiento de las 30 emisoras más bursátiles del mercado accionario mexicano, en función de las variaciones en los precios de las series accionarias y pesos relativos que tienen cada uno de los componentes que integran su muestra. Este es el primer Índice dentro de la familia de Índices de la BMV que incorpora en la determinación de los pesos relativos de su muestra variables operativas y fundamentales. (Bolsa Mexicana de Valores, S.A.B., 2019)

Este índice que está conformado por la "crema y nata" de las acciones que cotizan en la BMV, muestra el comportamiento de las 30 emisoras más bursátiles en la citada bolsa.

\section{GRÁFICA 33. S\&P/BMV BURSA OPTIMO EN CIFRAS CORRIENTES}

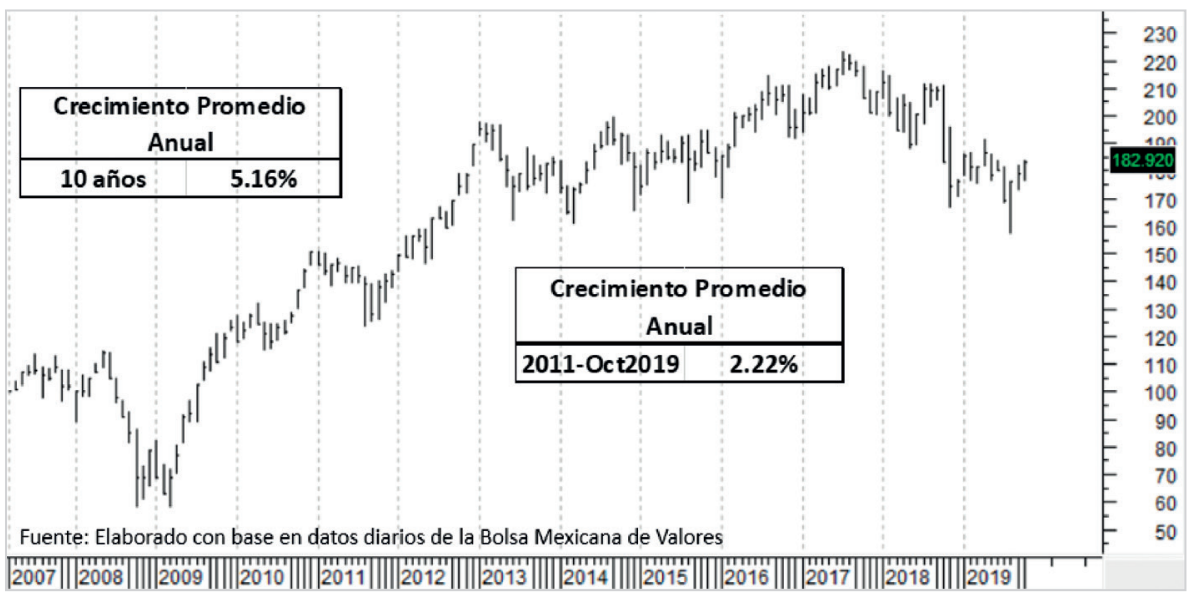

En la gráfica 33 se encuentra que el rendimiento promedio anual en 10 años del índice Bursa Optimo fue de 5.16\% y a partir del año 2011 y hasta el mes de octubre de 2019 de $2.22 \%$. 
GRÁFICA 34. S\&P/BMV BURSA OPTIMO EN CIFRAS CONSTANTES

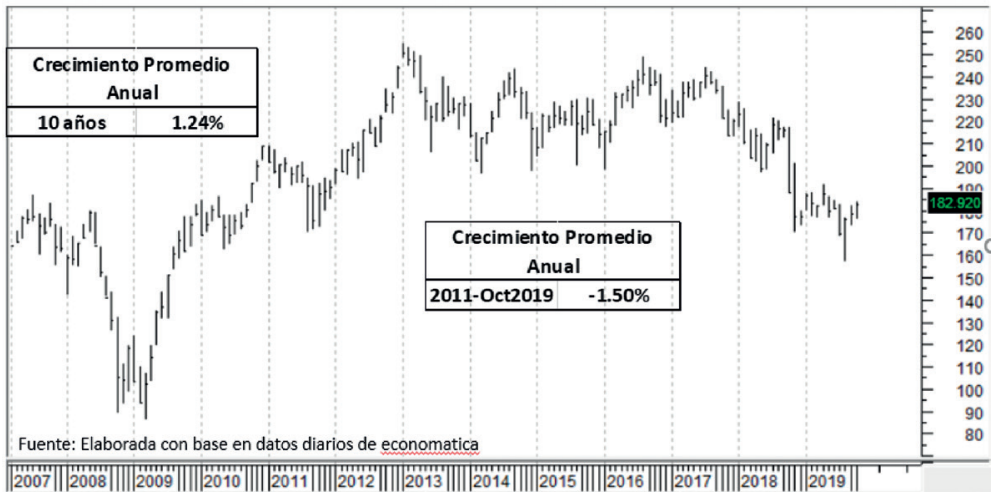

En cifras constantes, con rendimientos reales, la gráfica 34 muestra que la tasa de crecimiento de los 10 últimos años fue de $1.24 \%$ pero a partir del año 2011 y hasta el mes de octubre de 2019 el rendimiento fue negativo (-1.50\% promedio anual).

\section{GRÁFICA 35. S\&P/BMV BURSA OPTIMO EN USD}

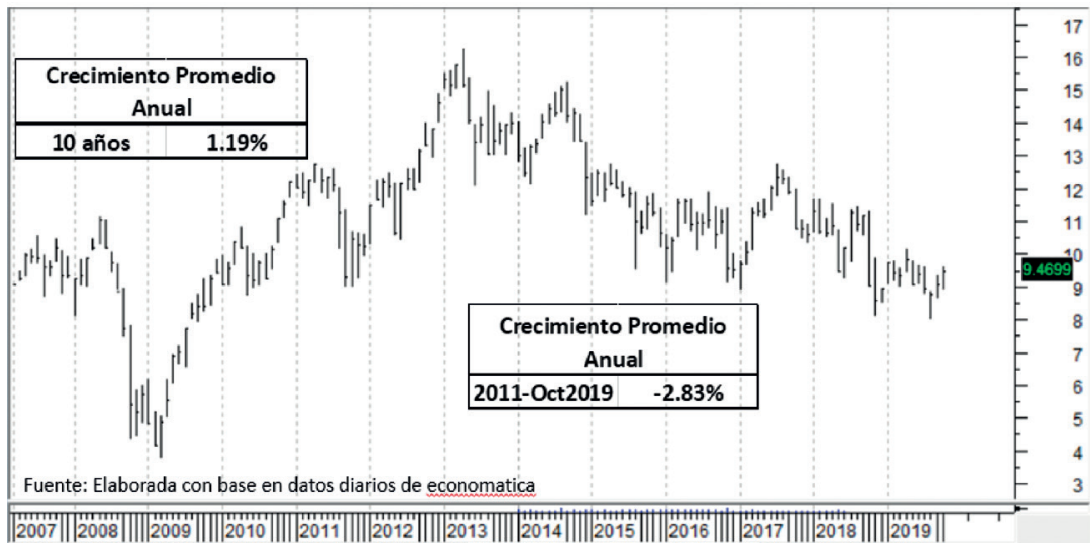

Valuando el rendimiento promedio anual en términos de dólares americanos, la gráfica 35 muestra que en 10 años se habría obtenido un rendimiento promedio anual de 1.19\%, sin embargo, si la inversión hubiera iniciado en el año 2011, el rendimiento hubiera sido de menos $2.83 \%$. 


\section{GRÁFICA 36. S\&P/BMV BURSA OPTIMO EN EUROS}

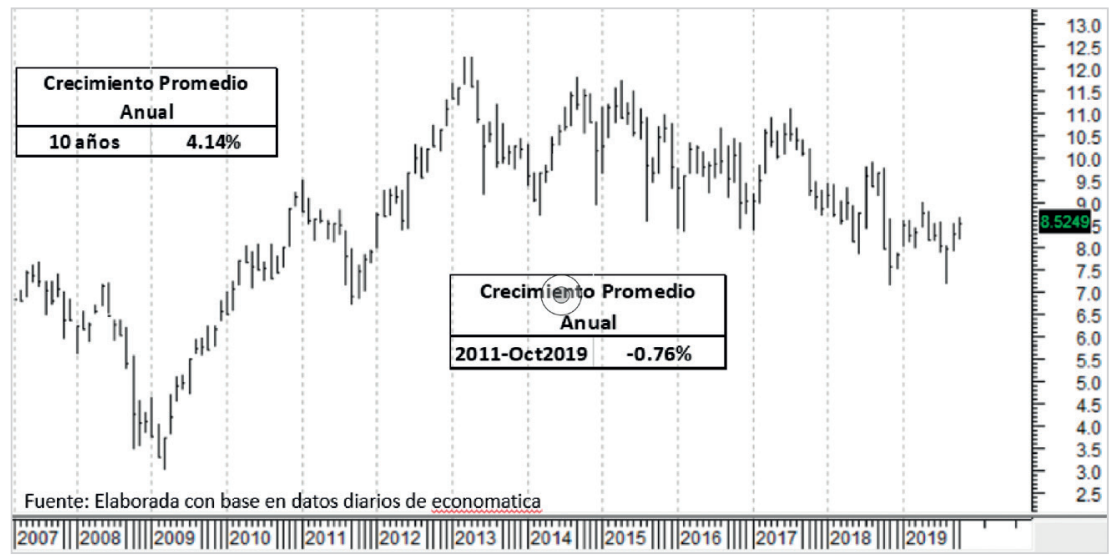

\section{Resultados obtenidos}

Se trató de analizar el comportamiento de diferentes índices de la BMV en un período de tiempo largo, 40 años y 30 años y en un período mediano, 10 años y del año 2011 al mes de octubre de 2019. Solo se pudo hacer con el S\&P/BMV IPC pues es el único índice del que se tienen datos históricos de esos años.

\section{CUADRO 1. RENDIMIENTO PROMEDIO ANUAL EN CIFRAS CORRIENTES}

\begin{tabular}{lccccc}
\hline $\begin{array}{c}\text { Índices Principales de la } \\
\text { Bolsa Mexicana de Valores }\end{array}$ & $\begin{array}{c}\mathbf{4 0} \\
\text { años }\end{array}$ & $\begin{array}{c}\mathbf{3 0} \\
\text { años }\end{array}$ & $\begin{array}{c}\mathbf{2 0} \\
\text { años }\end{array}$ & $\begin{array}{c}\mathbf{1 0} \\
\text { años }\end{array}$ & $\begin{array}{c}\mathbf{2 0 1 1} \mathbf{a} \\
\mathbf{3 1 - 0 c t - 1 9}\end{array}$ \\
\hline S\&P/BMV IPC & $29.90 \%$ & $16.90 \%$ & $10.92 \%$ & $4.23 \%$ & $1.33 \%$ \\
\hline S\&P/BMV INMEX RT & N.A. & N.A. & $12.48 \%$ & $6.18 \%$ & $3.25 \%$ \\
\hline S\&P/BMV IRT CompMx & N.A. & N.A. & N.A. & $7.02 \%$ & $4.09 \%$ \\
\hline S\&P/BMV IRT LargeCap & N.A. & N.A. & N.A. & $6.92 \%$ & $3.88 \%$ \\
\hline S\&P/BMV MidCap* & N.A. & N.A. & N.A. & $3.80 \%$ & $3.80 \%$ \\
\hline S\&P/BMV HABITA RT & N.A. & N.A. & N.A. & $-25.34 \%$ & $-28.69 \%$ \\
\hline S\&P/BMV FIBRAS** & N.A. & N.A. & N.A. & $3.71 \%$ & $3.71 \%$ \\
\hline S\&P/BMV IPC SUSTENTABLE & N.A. & N.A. & N.A. & $5.76 \%$ & $2.56 \%$ \\
\hline S\&P/BMV BURSA ÓPTIMO & N.A. & N.A. & N.A. & $5.16 \%$ & $2.22 \%$ \\
\hline
\end{tabular}

Fuente: elaborado con base en datos de la Bolsa Mexicana de Valores https://www.bmv.com.mx/\#

* 4 años ** 6 años 
Del Cuadro 1 destaca que el rendimiento en cifras corrientes decrece conforme el período de tiempo observado disminuye, esto únicamente en el período largo de tiempo en el S\&P/BMV IPC. En el período de 10 años y del año 2011 al mes de octubre de 2019 el índice que mayor rendimiento hubiera ofrecido fue el S\&P/ BMV IRT CompMX, que tiene por característica incluir en su muestra a las emisoras más grandes y más líquidas del mercado de valores mexicano. El segundo lugar en rendimiento lo hubiera obtenido el S\&P/BMV IRT LargeCap, que tiene como característica incluir en su muestra a las empresas más grandes.

CUADRO 2. RENDIMIENTO PROMEDIO ANUAL EN CIFRAS CONSTANTES

\begin{tabular}{llcccc}
\hline $\begin{array}{c}\text { Índices Principales de la } \\
\text { Bolsa Mexicana de Valores }\end{array}$ & $\begin{array}{c}\mathbf{4 0} \\
\text { años }\end{array}$ & $\begin{array}{c}\mathbf{3 0} \\
\text { años }\end{array}$ & $\begin{array}{c}\mathbf{2 0} \\
\text { años }\end{array}$ & $\begin{array}{c}\mathbf{1 0} \\
\text { años }\end{array}$ & $\begin{array}{c}\mathbf{2 0 1 1} \mathbf{a} \\
\mathbf{3 1 - o c t - 1 9}\end{array}$ \\
\hline S\&P/BMV IPC & $7.18 \%$ & $6.88 \%$ & $6.20 \%$ & $0.34 \%$ & $-2.35 \%$ \\
\hline S\&P/BMV INMEX RT & N.A. & N.A. & $7.68 \%$. & $2.23 \%$ & $-0.50 \%$ \\
\hline S\&P/BMV IRT CompMx & N.A. & N.A. & N.A. & $3.03 \%$ & $0.30 \%$ \\
\hline S\&P/BMV IRT LargeCap & N.A. & N.A. & N.A. & $2.80 \%$ & $-0.05 \%$ \\
\hline S\&P/BMV MidCap* & N.A. & N.A. & N.A. & $-0.38 \%$ & $-0.38 \%$ \\
\hline S\&P/BMV HABITA RT & N.A. & N.A. & N.A. & $-28.12 \%$ & $-31.29 \%$ \\
\hline S\&P/BMV FIBRAS** & N.A. & N.A. & N.A. & $-0.20 \%$ & $-0.20 \%$ \\
\hline S\&P/BMV IPC SUSTENTABLE & N.A. & N.A. & N.A. & $1.82 \%$ & $-1.17 \%$ \\
\hline S\&P/BMV BURSA ÓPTIMO & N.A. & N.A. & N.A. & $1.24 \%$ & $-1.50 \%$ \\
\hline
\end{tabular}

Fuente: elaborado con base en datos de Economatica

* 4 años ** 6 años

En el Cuadro 2 se observa que el único índice que logra obtener rendimiento positivo en el período del año 2011 al 31 de octubre de 2019 fue el S\&P/BMV IRT CompMx. En ese período de tiempo cualquier inversión que se hubiera mantenido en los otros índices habría destruido el ahorro invertido. Destaca de manera negativa la inversión en el índice S\&P/BMV HABITA RT, inversión en el mercado de vivienda, se habría reducido el capital a una tasa promedio anual de $-31.29 \%$. 
CuAdro 3. RENDIMIENTO PROMEDIO ANUAL EN USD

\begin{tabular}{llcccc}
\hline $\begin{array}{c}\text { Índices Principales de la } \\
\text { Bolsa Mexicana de Valores }\end{array}$ & $\begin{array}{c}\mathbf{4 0} \\
\text { años }\end{array}$ & $\begin{array}{c}\mathbf{3 0} \\
\text { años }\end{array}$ & $\begin{array}{c}\mathbf{2 0} \\
\text { años }\end{array}$ & $\begin{array}{c}\mathbf{1 0} \\
\text { años }\end{array}$ & $\begin{array}{c}\mathbf{2 0 1 1} \mathbf{a} \\
\mathbf{3 1 - 0 c t - 1 9}\end{array}$ \\
\hline S\&P/BMV IPC & N.A. & $9.40 \%$ & $7.17 \%$ & $0.34 \%$ & $-3.38 \%$ \\
\hline S\&P/BMV INMEX RT & N.A. & N.A. & $8.77 \%$. & $2.43 \%$ & $-15.70 \%$ \\
\hline S\&P/BMV IRT CompMx & N.A. & N.A. & N.A. & $7.17 \%$ & $2.34 \%$ \\
\hline S\&P/BMV IRT LargeCap & N.A. & N.A. & N.A. & $2.99 \%$ & $-1.13 \%$ \\
\hline S\&P/BMV MidCap* & N.A. & N.A. & N.A. & $0.32 \%$ & $0.32 \%$ \\
\hline S\&P/BMV HABITA RT & N.A. & N.A. & N.A. & $-27.82 \%$ & $-31.86 \%$ \\
\hline S\&P/BMV FIBRAS** & N.A. & N.A. & N.A. & $-2.80 \%$ & $-2.80 \%$ \\
\hline S\&P/BMV IPC SUSTENTABLE & N.A. & N.A. & N.A. & $1.84 \%$ & $-2.43 \%$ \\
\hline S\&P/BMV BURSA ÓPTIMO & N.A. & N.A. & N.A. & $1.19 \%$ & $-2.83 \%$ \\
\hline
\end{tabular}

Fuente: elaborado con base en datos de Economatica

* 4 años ** 6 años

Muchos ahorradores tienen preferencia por la inversión en dólares americanos y es por ese motivo que se analizó que habría sucedido si se hubieran convertido los diferente índices en términos de dólares. Nuevamente se confirma que el único índice que habría obtenido un mínimo rendimiento promedio anual positivo de más de $1 \%$ sería el S\&P/BMV IRT CompMx, 2.34\% promedio anual.

CuAdRo 4. RENDIMIENTO PROMEDIO ANUAL EN EUROS

\begin{tabular}{llllcc}
\hline $\begin{array}{c}\text { Índices Principales de la } \\
\text { Bolsa Mexicana de Valores }\end{array}$ & $\begin{array}{c}\mathbf{4 0} \\
\text { años }\end{array}$ & $\begin{array}{c}\mathbf{3 0} \\
\text { años }\end{array}$ & $\begin{array}{c}\mathbf{2 0} \\
\text { años }\end{array}$ & $\begin{array}{c}\mathbf{1 0} \\
\text { años }\end{array}$ & $\begin{array}{c}\mathbf{2 0 1 1} \mathbf{a} \\
\mathbf{3 1 - 0 c t - 1 9}\end{array}$ \\
\hline S\&P/BMV IPC & N.A. & N.A. & $6.87 \%$ & $3.35 \%$ & $-0.88 \%$ \\
\hline S\&P/BMV INMEX RT & N.A. & N.A. & $8.50 \%$. & $5.44 \%$ & $0.55 \%$ \\
\hline S\&P/BMV IRT CompMx & N.A. & N.A. & N.A. & $6.01 \%$ & $1.09 \%$ \\
\hline S\&P/BMV IRT LargeCap & N.A. & N.A. & N.A. & $6.02 \%$ & $1.00 \%$ \\
\hline S\&P/BMV MidCap* & N.A. & N.A. & N.A. & $0.18 \%$ & $0.18 \%$ \\
\hline S\&P/BMV HABITA RT & N.A. & N.A. & N.A. & $-25.73 \%$ & $-30.42 \%$ \\
\hline S\&P/BMV FIBRAS** & N.A. & N.A. & N.A. & $0.48 \%$ & $0.48 \%$ \\
\hline S\&P/BMV IPC SUSTENTABLE & N.A. & N.A. & N.A. & $4.79 \%$ & $-0.37 \%$ \\
\hline S\&P/BMV BURSA ÓPTIMO & N.A. & N.A. & N.A. & $4.14 \%$ & $-0.76 \%$ \\
\hline
\end{tabular}

Fuente: elaborado con base en datos de Economatica

* 4 años ** 6 años 
Algunos consideran, por diferentes razones, que es mejor invertir en euros y es por ese motivo que también se calcularon los resultados que se habrían obtenido en caso de invertir en algunos de los índices de la Bolsa Mexicana de Valores. Nuevamente estuvo el S\&P/BMV IRT CompMx en primer lugar con un rendimiento promedio anual de $1.09 \%$ y en este cálculo el S\&P/BMV IRT LargeCap también obtuvo un rendimiento promedio anual de $1.00 \%$.

Finalmente, respondiendo a la pregunta planteada al inicio:

- En la clasificación global de las Afores no hay ninguna clasificada como oro, solo una como plata y tres como bronce. No son las mejores.

- Las perspectivas económicas no son las mejores.

- Los resultados que ha ofrecido el mercado accionario, medido a través de los índices principales no son buenos si se miden en pesos constantes, en dólares americanos o en euros.

- Con base en esta evidencia podemos concluir que, de mantenerse esos resultados, el que inviertan en instrumentos de renta variable no colaborará en la obtención del objetivo de maximización de las carteras de inversión de las Siefores.

\section{Bibliografía}

Bolsa Mexicana de Valores, S.A.B. (31 de octubre de 2019). Tipos de índices.

Rappaport, A. (1986). Creating Shareholder Value. The New Standard for Business

Performance. Nueva York: The Free Press.

S\&P DOW JONES Indices. (31 de octubre de 2019). S\&P Dow Jones Indices.

Stewart, B. (1991). The Quest for Value. New York: Harper Collins.

Van Horne, J. C. (1977). Financial Management and Policy. New Jersey: Prentice-Hall. 